The abstracts are only available online, free of charge, under www.karger.com/journals/bpu/bpu_bk.htm

\title{
5th European Basic Multidisciplinary Hemodialysis Access Course
}

June 6-7, 2002, Brdo pri Kranju, Slovenia

\section{Abstracts}

Guest Editor

Marko Malovrh, Ljubljana, Slovenia

\section{Contents}

Basic Consideration

Abstracts 1-5

Access Creation

Abstracts 6-11

Vascular Access Complications - Aetiology and Diagnostics Abstracts 12-24

Other Complications and Types of Vascular Access Abstracts 25-30

Central Vein Catheter Access and Vascular Access Strategy Abstracts 31-36 


\section{Basic Consideration}

1

\section{No abstract received}

\section{2}

\section{Preservation of Veins in Pre-dialysis Patients and Early Referral}

\author{
V. Premru \\ Department of Nephrology, University Medical Center \\ Ljubljana, Ljubljana, Slovenia
}

Evaluation of patients with progressive renal failure for renal replacement therapy is complex. It includes a detailed medical history, physical examination, radiology/ultrasound etc. It should include evaluation of peripheral veins of upper extremities with venous mapping and evaluation of central veins. Veins should be preserved for future vascular access creation.

Some of the most important considerations for vascular access planning are:

1. history of: previous peripheral or central venous cannulations or venous catheters; pacemakers; arm, neck or upper chest surgery or trauma; diabetes mellitus; peripheral and coronary artery disease; heart failure; thrombotic events or other serious comorbidities; and/or

2. evidence of: upper extremity edema or differences in circumference; collateral veins; phlebitis/inadequate distensibility on tourniquet examination; scars from previous phlebotomies/catheter insertion/surgery; insufficient arterial flow or cardiac output.

Any of the above conditions might preclude successful creation of A-V fistula (AVF). Certain facts underscore the importance of vein preservation from the start in every patient with progressive renal failure: most patients with end-stage renal failure spend at least some time on hemodialysis (HD); distal (radiocephalic) native AVF is the best choice for vascular access followed by antecubital AVF; intact veins are needed to construct the native AVF; access problems have great impact on morbidity/hospitalization rate of patients on HD.

Venous preservation could be accomplished by adherence to the following principles:

1. Strict avoidance of cannulation of veins of both forearms proximal to the wrist.

2. When unavoidable, veinpuncture should be performed on the dominant arm to preserve the nondominant arm for AVF; or alternatively, rotation of puncture sites/sides could be used.
3. Phlebocatheters should not be threaded to central veins through cephalic or basilic veins at the elbow.

4. Instead, central vein catheters should be inserted into jugular veins (preferably on the right side).

5. Insertion via subclavian veins is to be avoided because of very frequent subsequent stenoses. The same applies to transvenously inserted pacemakers.

6. In cases where vein diameter/flow is the critical factor influencing decision to use the central vein (as when concentrated potentially caustic/toxic solutions are to be infused) one should consider using femoral veins.

This general strategy for venous preservation should be individually tailored to the above mentioned clinical findings and to the anticipated type/site of the planned access. Once established, the plan and strategy for AVF creation and venous preservation should be discussed with the patient. Patients should be well informed about the vital importance of well functioning vascular access for successful management on HD and the importance of vein preservation should be stressed. Patients should be instructed to refuse any veinpuncture at the unauthorized sites. Unfortunately, patients are generally not enough persuasive and many members of the medical personnel are still unfamiliar with this specific problem. Some additional solutions were developed to increase awareness on the matter: printed warning forms included in patient medical documentation, 'medical alert' cards and bracelets. Other educational measures for laboratory personnel, nurses and doctors should also be implemented.

Early referral for surgery is relevant for venous preservation as well. Patients should be referred for creation of AVF when their GFR falls below $25 \mathrm{ml} / \mathrm{min}$ or serum creatinine stays above approximately $400-450 \mu \mathrm{mol} / \mathrm{l}$. Early construction of AVF allows enough time for AVF to mature and avoids the need for temporary vascular access/ central vein catheter which by itself could damage vasculature. Also, in case of AVF failure this enables creation of a new AVF before the need for HD.

In summary, the first step to successful creation of vascular access is the initial evaluation and subsequent careful preservation of the vascular potential. Venous preservation is an important part of this goal. Early referral of the patient to nephrologist and thorough evaluation with multidisciplinary approach in preparation for and creation of vascular access is mandatory. Patients should be fully aware of the importance of the optimal vascular access for their future management. Additional education of medical staff involved in diagnostic and therapeutic procedures that demand venous cannulation/catheterization would also be appropriate. Special forms of medical warnings appear to be helpful. Careful planning of and timely referral for surgical creation of vascular access is another elementary demand to achieve the final goal: a well educated patient with optimal vascular access created in advance to enable most efficacious and uncomplicated dialysis management when the patient reaches end-stage renal failure.

\section{Literature}

1. National Kidney Foundation. K/DOQI Clinical Practice Guidelines for Vascular Access, 2000. Am J Kidney Dis 2001;37:S137-S181.

\section{KARGER}

(C) 2002 S. Karger AG, Basel 
2. Kumwenda M: Venous preservation; in Syllabus of the 1st Basic Multidisciplinary Hemodialysis Access Course, Paris, October 8-9th, 1998.

3. Pengloan J: Venous Preservation in Predialysis and Early Referral. Blood Purif 2002;20:(in press).

\section{3 \\ Clinical Examination Before Arteriovenous Fistula Construction}

\section{R. Ponikvar}

Department of Nephrology, University Medical Center

Ljubljana, Ljubljana, Slovenia

Preoperative patient's history and physical examination before arteriovenous fistula (AV) construction and before noninvasive Doppler mapping is an essential part of a patient's preparation for a surgical intervention.

Patient's history can reveal thrombotic events, heart disease with serious heart failure, heart surgery, anticoagulant or antiplatelet agents prescribed for various indications, low or high blood pressure, presence of diabetes mellitus or peripheral macroangiopathy, history of vascular accesses in the past (subclavian, jugular or femoral catheters, native of PTFE graft AV fistulas) and their function and complications related to them. All these information can help in planning future vascular access, focus further duplex-Doppler study on specific region and address specific questions for this valuable examination.

Physical examination should include inspection of infraclavicular and jugular sites for detecting the signs of previous catheters insertion, especially on the side of planned AV fistula construction. The presence of collateral veins on arm and/or asymmetrical arm edema indicating subclavian vein stenosis or occlusion is also recorded.

Normal veins of upper extremities are usually palpable and compressible after Tornique manouvre. If not, ultrasonogranography and/or phlebography are further steps in evaluating forearm and arm veins.

Normal arteries (without significant atherosclerotic changes) are usually easily palpable at the wrist and in the cubital fossa. If pulsations are strong, duplex-Doppler examination will not be strictly necessary. If no veins are detected clinically, that is strong indication to perform duplex-Doppler study and/or phlebography. If imaging studies reveals only concomitant veins, native $\mathrm{AV}$ fistula construction could not be performed.

Careful physical examination should be performed after native or PTFE graft AV fistula thrombosis. Size of thrombus formation as well as patent part of native/graft AV fistula should be assessed. If several days have been passed after thrombosis had occurred, the consistence of thrombus should have to be assessed in order to consider the degree of thrombus fibrosis and organisation which would influence the success of thrombolysis or thrombectomy.

Conclusions: Preoperative physical examination provides essential information about cardiovascular system, pre-existent native/graft $\mathrm{AV}$ fistula constructions, signs of central venous stenosis or occlusion and harvesting of saphenous veins for by-pass surgery. This examination should always proceed duplex-Doppler study, in order to address specific and focused questions for Doppler study making it as clinically usable and rational as possible. Physical examination is also the essential part in evaluating recently thrombosed native/graft AV fistula (before indicating eventual further imaging studies), necessary for optimal planning of salvage of thrombosed vascular access.

\section{4 Non-invasive Mapping of Vessels \\ M. Malovrh \\ Department of Nephrology, University Medical Center Ljubljana, Ljubljana, Slovenia}

Vascular access procedures and their subsequent complications represents a major cause of morbidity for chronic hemodialysis patients. Adequate blood flow through an arteriovenous fistula (AVF) is essential for adequate hemodialysis in the patients with end stage renal disease (ESRD). The most frequent problem with AVF are thrombosis and inadequate maturation. The risk factor for primary failure is not well established, although the quality of vessels is thought to play an important role. One of the most important predictors of successful AVF development is the ability of the venous and arterial vessels to dilate under the influence of the increased shear rates - vessel remodeling. The preoperative physical examination of the patients' forearm venous and arterial vessels include the quality of the arterial pulse and inspection of the vein with a tourniquet to induce venous congestion. Patients with advanced atherosclerosis and peripheral vascular disease such as diabetics, elderly and smokers are commonly found to have marginal vasculature. Duplex scanning is a promising method to establish certain morphological and functional parameters of peripheral blood vessels that is non-invasive and safe. This method has been recently used to visualize and measure arterial and venous vessel diameters, and has shown a good correlation between pre-operative determination and peri-operative findings. Routine preoperative sonographic vascular mapping results in increase of successful created AV fistulas. These improvements in outcomes are likely due to identification by ultrasound of suitable veins that were not apparent on physical examination. Many of the patients were found to have a large caliber veins that were simply too deep to be visualized or palpated at physical examination. In our study veins were clinically visible in 54/116 (46.5\%) and from 62/116 (53.5\%) veins were detected by ultrasound in $48 / 62(77.4 \%)$. The ability of the vein to dilate after AVF construction is also important for AVF patency after surgery and for adequate AVF maturation. To determine the ability of the vein to dilate properly increase of the internal vein's diameter after proximal vein compression and to show the continuity of the shape of Doppler vein signal (DVS) could be used. Outflow stenosis could be detected by pre-operative duplex sonography. To determine if there is a disturbance of venous outflow at increased venous return with deep breath (respiratory filling) could be used. In addition, Doppler waveform at the reactive hyperemia, induced by opening of the clenched fist for two minutes changes from threephasic high-resistance flow (peripheral resistance for peripheral arteries is normal high) to biphasic low-resistance flow (peripheral resistance become low for short time) and the same reaction is expected after AVF construction when the peripheral resistance for the radial artery feeding AVF decrease. In our study we have shown that lack of an appropriate reaction after releasing a fist indicates that even after AVF construction, the arterial blood flow not increase enough for AVF function. In our prospective study, resistance index at reactive hyperemia $\geq 0.7$ is indicator for AVF failure immediately after construction in almost $32 \%$ and internal diameter of artery $\leq 1.6 \mathrm{~mm}$ in $38 \%$ of 
constructed AVF. Higher resistance index and lower internal diameter of artery are associated with prolonged AVF maturation time.

In group of patients with marginal vessels expansion of blood volume could be used to increase blood flow through feeding artery and probably the occurrence of vasospasm could be prevented too. Group of patients with critical values (internal artery diameter IDA $<1.6 \mathrm{~mm}$, resistance index $\mathrm{RI}$ at reactive hyperemia $\mathrm{RH}>0.7$ and $\mathrm{ABF}<24 \mathrm{ml} / \mathrm{min}$ ) was divided in two groups by random sampling. One group received plasma expander during surgery and other not.

Total 102 AVF was constructed from Sept. 1999 to March 2001. At $58 \mathrm{pts}, 40$ males and 18 females, mean age $59.5 \pm 4.3 \mathrm{yrs}$, mean IDA was $2.8 \pm 0.4 \mathrm{~mm}, \mathrm{RI}$ at $\mathrm{RH}$ was $0.62 \pm 0.07, \mathrm{ABF} 44 \pm 9.2 \mathrm{ml} / \mathrm{min}$, $40 \mathrm{AVFs}$ was created at the wrist/forearm region and $18 \mathrm{AVFs}$ at the elbow region. Primary patency rate was $96.5 \%$ (56/58). At 44 pts, 28 males, 16 females, mean age $66 \pm 4$ years, mean IDA $1.9 \pm 0.4 \mathrm{~mm}$, $\mathrm{RI}$ at $\mathrm{RH} 0.83 \pm 0.3, \mathrm{ABF} 20 \pm 1.1 \mathrm{ml} / \mathrm{min}, 16$ AVFs was created at the wrist/forearm region and 28 at the elbow region. During surgical procedure $22 \mathrm{pts}$ mean age $60.6 \pm 1.7 \mathrm{yrs}, 14$ males, 8 females, IDA $1.8 \pm 0.5 \mathrm{~mm}, \mathrm{RI}$ RH $0.83 \pm 0.5,7$ wrist/forearm and 15 elbow region AVFs received mean $720 \mathrm{ml}$ (range 320 to $870 \mathrm{ml}$ ) of plasma expander (hydroxy aethyl starch). Patency rate in this group was $86.4 \%(19 / 22)$. In group of 22 pts mean age $62.8 \pm 3.2$ yrs, 13 males, 9 females, IDA $2.0 \pm 0.2 \mathrm{~mm}$, RI RH $0.87 \pm 0.6,9$ wrist/forearm and 13 elbow AVFs was created. During surgery they did not receive plasma expander. Patency rate was $27.3 \%(6 / 22)$. At 16 pts of this group plasma expander was given immediately after unsuccessful surgery in average $760 \mathrm{ml}$. Patency rate was $43.7 \%(7 / 16)$.

Non-invasive assessment of veins by duplex sonography is very helpful in patients with inadequate clinical vein visibility. In this way we can get also more information about functional characteristics of the veins and delineated venous outflow. Routine clinical evaluation is recommended to document adequacy of arterial inflow. When indicated, especially in elderly, patients with diabetic and analgesic nephropathy, vascular renal disease, and female patients, the clinician should also order a Duplex evaluation of the arteries. In this groups of patients it is important to create AVF early enough and based on the results of Duplex sonography the optimal site of AVF construction should be select. On the base of morphological and functional characteristics of the arteries determined by duplex sonography before surgery the need for expansion of blood volume could be predicted. The consequences of such procedure are less surgical interventions, earlier maturation of AVF, less stress for patients and at last but not least less money for AVF surgery.

\section{References}

1. Wedgwood AS, Wiggins PA, Guillou PJ: A prospective study end-to-side vs. end-to-end arteriovenous fistulas for haemodialysis. Brit J Surg 1984; 71:640-642.

2. Konner K: A primer on the av fistula-Achilles' heel, but also Cinderella of haemodialysis. Nephrol Dial Transplant 1999;14:2094-2098.

3. Konner K: Primary vascular access in diabetic patients: an audit. Nephrol Dial Transplant 2000;15:1317-1325.

4. Brescia MJ, Cimino JE, Appel K: Chronic hemodialysis using venepuncture and surgically created arteriovenous fistula. N Engl J Med 1966;275: 1089-1092.

5. Wong V, Ward R, Taylor R, Selvakumar S, How TV, Bakran A: Factors associated with early failure of arteriovenous fistulae for haemodialysis access. Eur J Vasc Endovasc Surg 1996;12:207-213.

6. Malovrh M: Non-invasive evaluation of vessels by duplex sonography prior to construction of arteriovenous fistulas for haemodialysis. Nephrol Dial Transplant 1998;13:125-129.
7. Lemson MS, Leiuinssen KLL, Tordoir JHM: Does preoperative duplex examination improve patency rates of Brescia-Cimino fistulas? Nephrol Dial Transplant 1998;13:1360-1361.

8. Girerd X, London G, Boutouyrie R, Mourad JJ, Safar M, Laurent S: Remodeling of the radial artery in response to a chronic increase in shear stress. Hypertension 1996;27:799-803.

9. Poredoš P, Kek A, Verhovec R: Morphological and functional changes of the arterial wall in subject at risk of atherosclerosis and in patients with peripheral arterial occlusive disease. 1997; Vasa26:271-276.

10. Lin SL, Huang CH, Chen HS, Hsu WA, Yen CJ, Yen TS: Effects of age and diabetes on blood flow rate and primary outcome of newly created hemodialysis arteriovenous fistulas. Am J Nephrol 1998;18:96-100.

11. Katz M, Camerona A, De Rojas J: B-mode imaging to determine the suitability of arms veins for primary arteriovenous fistulae. J Vasc Tech 1987; 9:172-174.

12. Rodriquez JA, Armadans L, Ferrer E, Olmos A, Codina S, Bartolome J, Borrellas J, Piera L: The function of permanent vascular access. Nephrol Dial Transplant 2000;15:402-408.

13. Hightower DR, Gooding GAO: Sonographic evaluation of the normal response of subclavian veins to respiratory monouvres. Radiology 1985; 20:517-520.

14. Savage T, Clarke AL, Giles M, Tomson CR, Raine AE: Calcified plaques is common in the carotid and femoral arteries of dialysis patients without clinical vascular disease. Nephrol Dial Transplant 1998;13:2004-2012.

15. Jungers P, Massy ZA, Khoa TN, Fumeron C, Labrunie M, Lacour B, Descamps-Latscha B, Man NK: Incidence and risk factors of atherosclerotic cardiovascular accidents in praedialysis chronic renal failure patients: a prospective study. Nephrol Dial Transplant 1997;12:2597-2602.

16. Yerdel MA, Kesenci M, Yazaicioglu KM, Doseyen Z, Turcapar AG, Anadol E: Effect of haemodynamic variables on surgically created arteriovenous fistula flow. Nephrol Dial Transplant 1997;12:1684-1688.

17. Reilly DT, Wood RFM, Bell PRF: Arteriovenous fistulas for dialysis: blood flow, viscosity, and long-term patency. World J Surgery 1982;6:628-633.

18. Lin SL, Chen HS, Huang, Yen T: Predicting the outcome of hemodialysis arteriovenous fistulae using duplex sonography. J Formos Med Assoc 1997; 96:864-868.

19. Anderson CB, Etheredge EE, Harter HR, Codd JE, Graff RJ, Newton WT: Blood flow measurements in arteriovenous dialysis fistulae. Surgery 1977; $81: 459-461$

20. Silva MB Jr, Hobson RW 2nd, Pappas PJ, Jamil Z, Araki CT, Goldberg MC, Gwertzman G, Padberg FT Jr: A strategy for increasing use of autogenous hemodialysis access procedures: impact of preoperative noninvasive evaluation. J Vasc Surg 1998;27:302-307.

21. Either JH, Lindsay RM, Barre PE, Kappel JE, Carlisle EJ, Common: Clinical practice guidelines for vascular access. J Am Soc Nephrol 1999; (suppl 13)10:S297-S305.

22. NKF/DOQI Clinical practice guidelines for vascular access: update 2000. Am J Kidney Dis 2001(suppl 1);37:S137-S181.

23. Allon M, Lockhart ME, Lilly RZ, Gallichio MH, Young CJ, Barker J, Deirhoi HM, Robbin LM: Effect of preoperative sonographic mapping on vascular access outcome in hemodialysis patients. Kidney International 2001;60:2013-2020.

24. Malovrh M: Native arteriovenous fistula: preoperative evaluation. Am J Kidney Dis 2002;39:(in press).

\section{5}

\section{Phlebography Before Angioaccess Creation}

\section{A. Raynaud \\ Hôpital Européen Georges Pompidou, Clinique Alleray-Labrouste, Paris, France}

Before creating an angioaccess, patency and quality of superficial and proximal veins are difficult to evaluate. Their assessment is based on clinical examination, ultrasonography, nuclear magnetic resonance 
imaging and overall history and phlebography. Regular phlebography requires injection of iodine contrast material, which is contra-indicated in cases of renal failure. Thus, indications for iodine phlebography are very wide in patients who are already hemodialysed and very limited in patients with severe renal failure who have not been previously dialysed.

Major value of phlebography is avoiding attempting to create a fistula on a vein which is not suitable for access creation or to detect a clinically unsuspected suitable vein. Phlebography should satisfactorily visualise all the superficial veins in the upper limb and the deep central veins.

The main indications for phlebography are:

- insufficient clinical examination;

- past history of central vein line placement which may have compromised patency of proximal veins;

- failure to create a first access.

\section{lodine Phlebography of the Upper Limb}

Vein puncture should be performed on a vein of the dorsum of the hand. The upper limb should be placed in anatomic position (supine position) in order to allow identification of the veins.

Opacification: All the superficial veins of the upper limb should be filled by the dye, and not only those located on the direct run off the puncture site. The deep central veins should also be visualised. A large amount of contrast material is therefore required. In order to reduce the amount, the dye can be pulsed by $5 \%$ glucose solution. We routinely use 35 to $40 \mathrm{cc}$ of dye pulsed by 40 to $50 \mathrm{cc}$ of $5 \%$ glucose solution.

Veins should be distended to allow evaluation of their real size and quality. This can be achieved by using a tourniquet placed on the arm at least a few minutes before injection. However a tourniquet encourages filling of the deep veins and may lead to non-visualisation of the cephalic vein in the arm. We therefore recommend removing it when $2 / 3$ of the dye has been injected. Another factor which favours vein distension is injection of a large volume of fluid upstream from the tourniquet. Occasionally injection of nitroglycerine is helpful when differentiation of spasm from real venous lesion is not possible. Nitroglycerine should be injected while the tourniquet is tightened. This increases the time of contact between vein walls and the drug and thus improves its efficacy. The drug we use is molcidomine ( $0.5 \mathrm{mg}$ per limb diluted in $20 \mathrm{cc}$ of saline solution).

Some false images of stenosis should be borne in mind:

- despite removal of the tourniquet its print on the cephalic vein often remains during image acquisition.

- in anatomic position compression by the soft tissue can cause false stenosis or occlusion of the deep vein at the brachio-axillary junction.

- wash out due to non-opacified flow coming from cerebral veins may cause a false image of stenosis.

\section{Selective Phlebography}

The aim of selective phlebography is to define the quality of one vein segment. This examination is requested when the clinical examination shows a vein but there is some doubt about the quality of one segment of this vein.

The phlebography technique is then completely different. The puncture site should not be located on veins potentially suitable for access creation but on one of its roots. Venous dilatation by tourniquet with or without nitroglycerine is often required. When digital subtraction angiography is used low amount of highly diluted iodine contrast material (up to $90 \%$ ) can be injected. Severe renal failure does not then contraindicate this examination which can be achieved with less than $2 \mathrm{cc}$ of dye.

\section{$\mathrm{CO}_{2}$ Phlebography of the Upper Limb}

It can be performed in case of renal failure and of allergy to iodine contrast material. This examination can thus easily be attempted before angioaccess creation. The technique of $\mathrm{CO}_{2}$ phlebography in our institute is close to that of iodine. However, frames should be acquired at a higher rate (6 images/s) and filling of superficial veins is more difficult to obtain. Nitroglycerine injection is therefore systematically required.

We inject $\mathrm{CO}_{2}$ manually using a $50 \mathrm{cc}$ syringe. Injection of $\mathrm{CO}_{2}$ at a satisfactory rate therefore requires an experienced operator.

$\mathrm{CO}_{2}$ phlebography is well tolerated by patients, although they often complain of dizziness during the 30 minutes following the examination. We therefore keep the patient lying down for half an hour after this examination.

Major complications are mainly due to over-injection of $\mathrm{CO}_{2}$, which may even cause patient death. This occurs when a stop cock remains open and the $\mathrm{CO}_{2}$ goes directly from the cylinder to the patient. To avoid this complication, we only open the tap of the $\mathrm{CO}_{2}$ cylinder for a few seconds to fill the pressure reducer. All the examinations are then performed with the $\mathrm{CO}_{2}$ contained between the two manometers of the pressure reducer.

When the cephalic veins are not sufficiently opacified in the forearm, another injection with compression of a superficial vein draining the $\mathrm{CO}_{2}$ may be indicated.

When the cephalic vein is not opacified in the upper arm because of preferential opacification of the basilic vein, a new injection should be performed with compression of the basilic vein. Placing the arm along the body is often sufficient to squash the basilic vein at the humero-axillary junction. In lean patients this manoeuvre should be reinforced by placing a hard object in the axillary fossa. However a new injection with the arm in abduction may be indicated to opacify the basilic vein in obese patients.

In our experience, the results of $\mathrm{CO}_{2}$ phlebography are similar to those of iodine phlebography. Allowing satisfactory visualisation of superficial upper arm veins of central veins.

\section{Access Creation}

\section{6}

\section{Forearm AVF: Strategy and Microsurgical Procedures}

\section{P. Bourquelot \\ Clinique Jouvenet, Paris, France}

We started using microsurgery in children and in adults for the creation of distal AVF in the late seventies, and preventive hemostasis in the early nineties [1]. 
In children our results for AVF microsurgery under $10 \mathrm{~kg}$ were published in 1981 [2]. In 1990 [3] we reported 380 children receiving microsurgery for AVF: the immediate patency rate was $96 \%$ and the 24 -month patency rate was $85 \%$ in distal radial-cephalic AVF. The benefit of microsurgery for children was emphasised in 1984 by Yazbeck [4], in 1993 by Sanabia [5] and in 1998 by Bagolan [6]. In adults also (Lim, Margic, Luchian) microsurgery in angioaccess gaining is a great benefit for the patient and a valuable teaching instrument too.

Conservative use of the venous system is a must in every patient doomed to hemodialysis. When possible, the dorsal veins of the hand must be used for blood sampling. Absolute respect of the nondominant arm is necessary. Subclavian central venous catheters with high risk of proximal vein stenosis are prohibited.

Careful preoperative clinical examination and Doppler examination assess the condition of the vein; a venous angiography (iodine or $\mathrm{CO}_{2}$ ) is necessary if there is any doubt, particularly when central venous catheter has been inserted previously.

Prophylactic broad-spectrum perioperative antibiotic is prescribed.

The rules for suturing are: forceps must never grasp the intima, the adventitia is incised, not resected, high pressure clamps must be avoided, the thinnest possible needles are to be used.

Preventive hemostasis, using a pneumatic tourniquet, makes extensive arterial dissection for clamping unnecessary: arterial spasm and ischemia are avoided. When hemostasis is incomplete, microclamps are placed on the artery after minimal dissection.

\section{Various AVF}

\section{Radial to Cephalic AVF}

The cutaneous incision is longitudinal, half way between the cephalic vein and the radial artery in the wrist. The nerves must be carefully preserved. The vein is freed by a wide dissection, a ligature being placed on the collateral branches: this avoids the use of an electric scalpel that could damage the vascular trunk itself. The diameter of the vein is measured. The vein is irrigated frequently in order to prevent drying and it is only handled by its adventitia. After section of the vein above a distal ligature, posterior incision of approximately $10 \mathrm{~mm}$ in length is made in the proximal vein. The anterior wall of the artery is exposed, without dissecting the arterial trunk.

A longitudinal arteriotomy is commenced with a disposable ophthalmologic scalpel, taking great care not to damage the posterior wall. This is completed with scissors. If a few drops of blood appear they are rinsed with heparin saline and the blood is dried with small sponges. The anastomosis is made with four running sutures, by suturing first the proximal angle.

It is preferable to keep the running suture untightened at the beginning so as to leave the lumen of the artery open. Frequent use of the zoom facilitates this very precise part of the operation; of course the needle must be repositioned in the needle holder for each passage through a vessel. Both proximal running sutures (posterior and anterior) are stopped at the middle of the arteriotomy.

The rest of the anastomosis is made in starting from the distal angle. The tourniquet is then released. If there is a leak, an additional suture is added. Patency is confirmed by Doppler ultrasound. The murmur can be absent during the first post-operative hours particularly in small children. It is very important to ascertain that the vein is not stenosed at the upper limit of the dissection.

\section{Variations}

Two running sutures anastomosis. The proximal suture is made first, starting at the middle of the posterior wall of the arteriotomy, going up to the proximal angle, and down to the middle of the anterior wall. The distal suture is made afterward. This technique gives an excellent vision on the arterial lumen.

Transposition. It is sometimes necessary to surgically transpose subcutaneously the vein two months later in small children with thick adipose tissue or in obese patients. An interval of three weeks must be respected before puncturing.

\section{Ulnar to Basilic AVF}

A sufficient length of the vein, which is situated quite far back from the artery, must be freed. It can be a help to put the forearm in flexion at the elbow during dissection. The artery is approached by passing behind the flexor carpi ulnaris, which can be transected. The interval before puncture is longer than with a radial to cephalic AVF.

\section{Strategy}

- All patients must have a microsurgical radial to cephalic or ulnar to basilic distal AVF, if possible, created one month before use ( 2 to 3 months in children). In children under $10 \mathrm{~kg}$ the delay is longer.

- Arterio-venous grafts, still recommended by a few authors, must be avoided at all costs in children [7] in view of the rapidity with which they become complicated by stenosis of the venous anastomosis. In adults, graft is a second choice after distal and proximal AVF.

- In case of emergency and if peritoneal dialysis is impossible, a central venous catheter must be inserted. A subclavian catheter is to be avoided because of the risk of interference with the venous drainage of the limb with dramatic consequences for the future. An internal jugular vein catheter is preferable.

Microsurgery has greatly improved and simplified the creation of distal AVF. In adults long term patency of distal AVF is observed frequently, up to 25 years. In children microsurgery has virtually eliminated the use of long term venous catheters and arterio-venous grafts.

\section{References}

1. Bourquelot P: Preventive haemostasis with an inflatable tourniquet for microsurgical distal arteriovenous fistulas for haemodialysis. Microsurgery 1993;14:462-463.

2. Bourquelot P, Wolfeler L, Lamy L: Microsurgery for haemodialysis distal arteriovenous fistulae in children weighing less than $10 \mathrm{~kg}$. Proc Eur Dial Transplant Assoc 1981;18:537-541.

3. Bourquelot P, Cussenot O, Corbi P, Pillion G, Gagnadoux MF, Bensman A, Loirat C, Broyer M: Microsurgical creation and follow-up of arteriovenous fistulae for chronic haemodialysis in children. Pediatr Nephrol 1990;4: 156-159.

4. Yazbeck S, O'Regan S: Microsurgery for Brescia-Cimino fistula construction in pediatric patients. Nephron 1984;38:209-212.

5. Sanabia J, Polo JR, Morales MD, Canals MJ, Polo J, Serantes A: Microsurgery in gaining paediatric vascular access for haemodialysis. Microsurgery 1993;14:276-279.

6. Bagolan P, Spagnoli A, Ciprandi G, Picca S, Leozappa G, Nahom A, Trucchi A, Rizzoni G, Fabbrini G: A ten-year experience of BresciaCimino arteriovenous fistula in children: technical evolution and refinements. J Vasc Surg 1998;27:640-644.

7. Bourquelot P, Gagnadoux MF: Vascular access for hemodialysis in children. Pediatr Nephrol 1997;11:659-660. 


\section{7}

\section{Elbow and Upper Arm Native Fistulas}

\section{Malovrh}

Department of Nephrology, University Medical Center

Ljubljana, Ljubljana, Slovenia

During the first decade after beginning of treatment of patients with ESRD by chronic haemodialysis generations of nephrologists and vascular surgeons were taught that the success of vascular access depended only on availability of a suitable peripheral veins at the wrist or forearm. The increasing proportion of older and diabetic patients in the haemodialysis population increasing also the problem of feeding arteries for arteriovenous fistula (AVF) as the most frequent vascular access.

Atherosclerosis is primarily an intimal disease whereas arteriosclerosis is primarily a medial degenerative condition causing dilatation, diffuse hypertrophy and stiffening of arteries. The development of atherosclerosis in uraemic patients is seen in the earliest stages of chronic renal failure and progresses relentlessly on dialysis. These observations shifted a focus from the veins to the arteries, particularly to the quality of the arterial wall and the diameter of the arterial lumen. An atherosclerotic and calcified radial artery of the wrist or distal part of the forearm will deliver only a limited blood flow rate and will not undergo adaptive flow-mediated dilatation to deliver sufficient fistula blood flow. Arterial calcifications are progressively less from the wrist to the elbow region. This consideration led the different authors to the idea of using proximal radial artery or the brachial artery in the region of the elbow or the upper arm when constructing a first AVF in the diabetic or older patient.

Before the decision for one of those location of AVF construction assessment of a variety of clinical parameters should be performed: medical history-diabetes, hypertension, peripheral ischaemia etc., careful search for suitable vein, quality of the arterial pulses along the radial, ulnar, and brachial arteries, ultrasonographic evaluation of the arterial and venous system in both arms. Doppler investigation has recently become an essential part of preoperative diagnostic procedure, providing information on diameter and wall structure of the artery as well as arterial blood flow rates. Besides the problem with artery there still could be the problem with suitable veins at the arm.

Types of anastomosis-technical consideration: Since 1968 artery side to vein end anastomosis is very popular and is much more effective than artery end to vein end. There was many studies showed that about 25 to $40 \%$ of AVF flow is coming from the distal part of the artery and there are some authors showed that if distal part of artery was thrombosed immediately after AVF construction, AVF failed very early. There is one more reason for such type of anastomosis. In diabetics and elderly patients with vascular problems the peripheral disruption of the radial artery could be very risky. Side of artery to side of vein is very rare anastomosis because of great risk for venous hypertension at the distal part of the arm and the AVF flow could be too small for effective haemodialysis treatment.

Location: construction of an arteriovenous anastomosis is possible at various locations, depending on the quality of vein and artery. Construction of AVF in the elbow region offers a series of possibilities with regard to location and the type of anastomosis, depending on the individually varying arrangement of the native vessels, mainly of the venous network. In most cases the anastomosis will result in arterialization of the cephalic as well of the basilic vein at the upper arm. Because of superficial position, the cephalic vein can be cannulated easy for many years. The first superficial part of the basilic vein in the region of elbow is very short in some patients. But if there is suitable cephalic vein and also a short part of the basilic vein the punctures of needles could be very near and there will be no recirculation. Some times the artery in elbow region is also very atherosclerotic. That means that the structure of the vessel wall will not allow dilatation, the prerequisite for sufficient arterial inflow and the high blood flow rates needed for haemodialysis treatment. Brachial artery in elbow region has internal diameter twice as is internal diameter of radial artery and this can compensate bad quality of vessel wall. Sometimes cephalic vein in the elbow region is missed and in this case the cephalic vein can be mobilised from the lateral aspect of this region and sutured to brachial artery. When cephalic vein distal of the venous triangle in the elbow region is phlebitically changed or even thrombosed, so called Gracz AVF could be performed. The perforating vein, originating from various part of venous triangle is anastomosed to the brachial or to the beginning of radial artery in this region. The length of anastomosis is limited by the diameter $3-5 \mathrm{~mm}$ of perforating vein and by this there is reduction of the risk of peripheral ischaemia. The anastomosis is buried in the depth of the elbow and so protected from accidental arterial puncture while placing the dialysis needles. In the study of Konner, 347 primary AVF was created, at $145(41.8 \%)$ the elbow region was the primary choice, in 87 $(32.3 \%)$ non-diabetics and in $58(74.7 \%)$ of diabetics.

Another problem will arise in cases when cephalic vein on the upper arm is not available: the first segment of basilic vein in the region of elbow is too short in many patients, thus providing only a limited stretch for cannulation and there is a great possibility of recirculation. The superficialization of the basilic vein along the medial aspect of the upper arm can give good long-term results. Longitudinal skin incision is performed from the elbow region to the axilla. The basilic vein is cut off in the elbow and anastomosis to the brachial artery is performed, normally the length is the same as internal diameter of vein. After releasing blood flow through fistula vein, basilic vein is mobilised under the subcutaneous tunnel $2-3 \mathrm{~cm}$ away from the skin incision and fixed by a few sutures. In this position, AVF vein after 4-6 weeks is within easy reach for cannulation. Subsequent studies have shown that upper arm AVF can be used effectively to increase the prevalence of native fistulae, even in women, older patients and those with diabetes, for whom lower arm AVF is difficult to establish.

Another method is so called 'two step AVF construction'. First step is anastomosis of basilic vein to the brachial artery and after few weeks superficialization of basilic vein and transposition like in the first type of method. By our experiences the results are almost the same with the exception of two operations needed in the second method and because of that longer maturation time.

In conclusion, the aim of the surgeon is to create a well functioning AVF. The artery and the vein have to be "healthy, that means that the structure of the vessel wall will allow dilatation, the prerequisite of sufficient arterial inflow and the high fistula blood flow needed for haemodialysis treatment'. In given individual, for the location of the first AVF one has to select a segment where artery has good wall quality or even internal diameter is great enough to ensure useful blood flow. An increasing proportion of patients is seen nowadays in whom a first AVF has been successfully created in the elbow region or even on the upper arm. Elbow and upper arm AVF are indicated in all patients with exhausted peripheral venous network. 


\section{References}

1. Konner K: When insufficient arterial inflow becomes the Achilles heel of the av-fistula-what are the surgical approaches? Nephrol Dial Transplant 2000;15:145-147.

2. Sedlacek M, Teodorescu V, Falk A, Vassalotti JA, Uribarri J: Hemodialysis access placement with preoperative noninvasive vascular mapping: comparison between patient with and without diabetes. Am J Kidney Dis 2001;38:560-564.

3. Dixon BS, Novak L, Fangman BS: Hemodialysis vascular access survival: upper-arm native arteriovenous fistula. Am J Kidney Dis 2002;39:92-101.

4. Konner K: Primary vascular access in diabetic patients: an audit. Nephrol Dial Transplant 2000;15:1317-1325.

5. Konner K: A primer on the av fistula-Achille's heel, but also Cinderella of haemodialysis. Nephrol Dial transplant 1999;14:2094-2098.

6. Berardinelli L: Upper arm arteriovenous fistula using native vessels. Blood Purif 2002;20:(in press).

7. Windus DW: Permanent vascular access: A nephrologist's view. Am J Kidney Dis 1993;21:457-471.

8. Hakaim AG, Nalnabndian M, Scott T: Superior maturation and patency of primary brachiocephalic and transposed basilic vein arteriovenous fistulae in patients with diabetes. J Vasc Surg 1998;86:211-216.

9. Kalman PG, Pope M, Bhola C, Richardson R, Sniderman KW: A practical approach to vascular access for hemodialysis and predictors of success. J Vasc Surg 1999;30:727-733.

08

\section{Lower Limb A-V Fistulas}

J. Barbosa*, F.M. José**

* Hospital Santa Maria, Lisboa, Portugal

**Hospital Garcia De Orta, Almada, Portugal

\section{Introduction}

An increasing number of patients with chronic renal failure are requiring long-term dialysis and the maintenance of functional vascular access is one of the most important and difficult problems for surgeons enveloped with this type of pathology.

A large number of these patients have already exhausted the great majority of the upper extremity vessels, reason why the lower limbs, presenting vessels with large calibre, are a good alternative for creation of new angioaccess.

The lower limb A-V fistulas, presents a higher rate of patency, comparing with those performed in other anatomic regions, fact that can be explained for the higher flow of these fistulas.

During the last year, no significant contributions to this subject were published in the international literature, reason why we based this abstract, with few modifications, in the one we presented last year, concerning a very similar theme - 'Femoral-bridge grafts'.

\section{Lower Limb A-V Fistulas}

Classically, the lower limb AVF grafts were introduced as second alternative when exhausted the more desirable upper extremity vessels.

However, as they reveal a higher flow due to the larger vessels when compared with the upper limb grafts, they are actually the first choice in severally hypotensive patients.

Another polemic indication for this access as a first option is the aesthetic or functional preservation of the upper limb for some type of professions. For some authors, this type of graft presents the advantage of facilitating the self-cannulation, because the upper limbs are free.
Pre-operative evaluation, should include an arterial and venous investigation of the limb. When the distal pulses in the foot are not detected, the determination of ankle-brachial pressure index is mandatory.

If this index is lower than 0.75 or if the patient has claudication, the risk of limb ischemia by a steal phenomenon requires further evaluation, sometimes including an angiography. These are cases for a personalised evaluation, based in the available options for access.

Contraindications for lower limb access are the cases of critical chronic ischemia of the lower limb, including class III and IV of Leriche-Fontaine.

Occlusions or stenoses of the iliac or femoral vein are other relative contra-indication for this surgery. Sometimes, they are not clinical evident and the ultrasonography Doppler can help in defining their permeability. Associating the endovascular procedures is possible to implant grafts after dilatation of an iliac vein, stenosed by a previous catheter.

Based in these principles, our preferred technique in the thigh is the femoral superficial artery-saphenous vein, loop graft. (fig. 1, 2) This configuration permits the sparing of the origin of the femoral profunda artery and the collateral derived from it that provide the

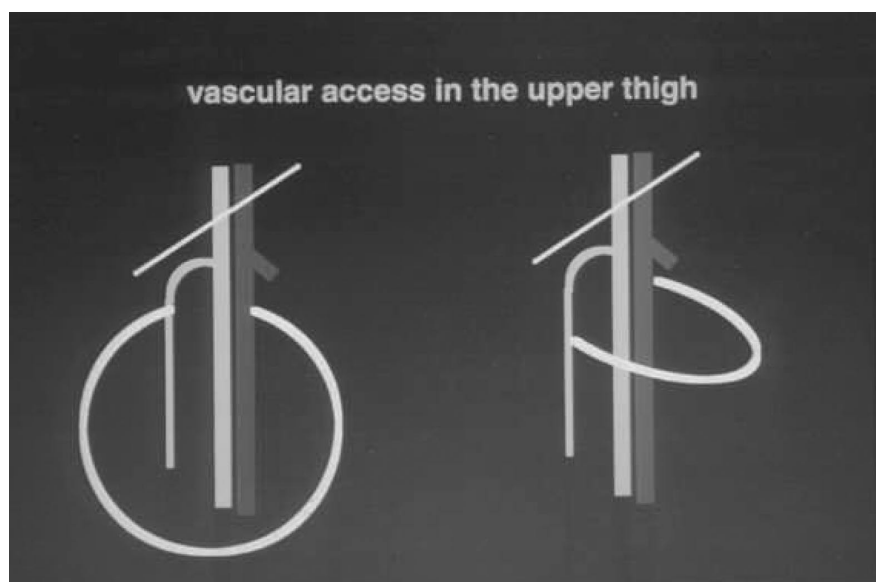

Fig. 1.

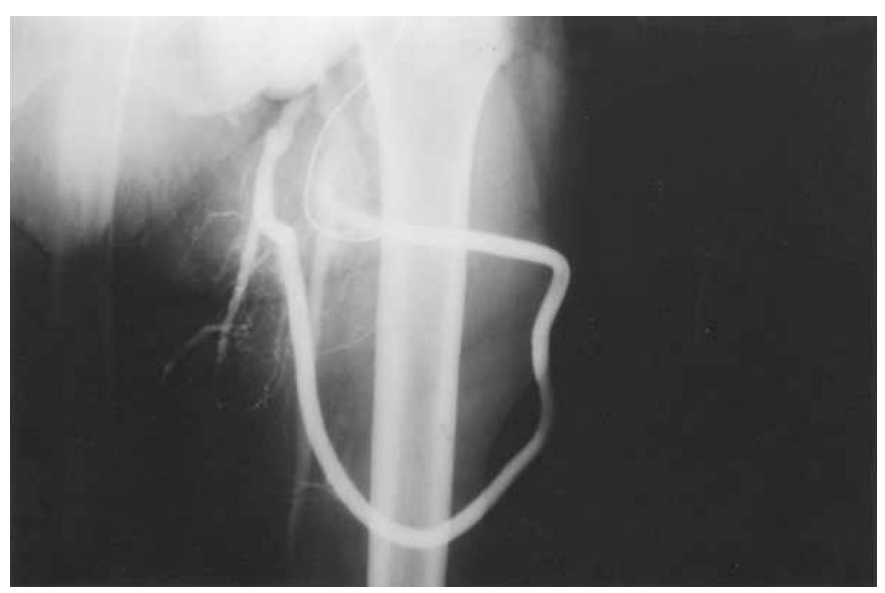

Fig. 2. 
limb in cases of ischemia. The graft is tunnelled laterally in the thigh, permitting an easy cannulation and commodity for the patient. This also permits a needle puncture in an area far from the contaminated region of the groin, for preventing infection.

The superficial femoral artery-saphenous vein grafts also possibilities the use of proximal vessels, common femoral artery and vein for a construction of a new access, when the previous fails.

Other alternatives were used in our experience, based in the patent vessels and the previous grafts. Saphenous vein, femoral, iliac or even vena cava were used in combination with the ipsilateral artery like the popliteal, superficial, common femoral or iliac. In most cases the loop configuration in the tight was used.

In some cases we perform a crossover graft from the artery to the opposite limb vein, with very acceptable results.

Multiples are the surgical techniques that has been used:

- Sup. Fem. A. - Saph. V. - loop graft

- Com. Fem. A. - Saph. V. - loop graft

- Sup. Fem. A. - Saph. V. - 'interposition' graft

- Com. Fem. A. - Ext. Iliac. V. - loop graft

- Com. Fem. A. - Vena Cava - loop graft

- Sup. Fem. A. - Saph. V. - loop AVF

- Popl. A. - Saph. V. - AVF

- Post. Tibial A. - Saph. V. - AVF

Today we support the idea that P.T.F.E. presents better long terms results, comparing with the vein, reason why we try to preserve the saphenous vein for other type of angioaccess.

Concerning the anastomosis, a continuous 6/0-prolen suture has been, usually, performed.

The rate of complications of the AVF in the lower limbs, was similar to the grafts of other localisation, including infection and false aneurysms.

Some authors had described a high incidence of infection in the femoral grafts when comparing with those performed in the upper limbs, and this was an important argument against this specific localisation. However, our personnel experience, correlated with posterior series, demonstrated that the rate of infection was not superior of the upper limb grafts.

\section{Summary}

The lower limbs AVF should be performed for vascular access, normally, as a second alternative.

The desperate cases in which are exhausted all the vessels in the upper limb, are the major indication for grafts with this localisation.

However, when we need a high blood flow fistula, such as in elderly patients with severe hypotension they can be a first option. Concerning technical problems, the ideal graft should be placed in loop, laterally in the thigh, between the superficial femoral artery and the long saphenous vein. So we can avoid ischemic complications of the limb and permit new surgical alternatives, based on the principles that each dialysis site should be preserve for use, as long as possible. The rate of complications of femoral bridge grafts, specially the infection, is not higher comparing with grafts placed in the upper limbs. The major contraindication for femoral angioaccess is the atherosclerotic occlusive diseases of the lower limbs, principally, in the more advanced stages. The lower limb AVF constitute a valid alternative in the therapy of chronic renal patients in hemodialysis, if respected the correct indications and the technical aspects we mentioned.When we looked for the patency, the femoral access presented a higher rate comparing with other type of access, fact that can be explained for the higher flow of the shunt, due to the larger vessels with more favourable hemodynamics.

Other complications of the access like steal syndrome and leg oedema can be seen. The first is, normally, related with atherosclerotic obstructions of the lower limb arteries and this happens in desperate cases, when we have to create an access in a potentially ischemic limb, because there is no other option available. Leg oedema is frequently seen after arterio-venous femoral fistulas, but in most cases is mild to moderate. This is due to the different hemodynamics of veins of the lower limbs that are not prepared to stand the higher pressure, against gravity. In the treatment of the complications, reconstructive conventional surgery was the adopted therapy in the great majority of cases. In the last five years, the number of femoral bridge grafts performed is progressively decreasing. This fact is supposed to be related with a much better planning of the previous vascular access, in our country. The lower limb AVF represent, in our days, a very important alternative in the surgery of the vascular access for hemodialysis. This type of surgery, specially dedicated to desperate cases is strongly supported by the creative imagination of the surgeon, more than any other.

9

\section{PTFE Graft}

J.R. Polo

Vascular Access Unit, Hospital General Universitario

Gregorio Marańon, Madrid, Spain

\section{Indications}

A graft for dialysis should be placed only when an forearm or upper arm autologous fistula cannot be performed due to lack of adequate superficial veins. Due to the high incidence of grafts complications, peritoneal dialysis should be considered before a graft is constructed.

Prospective studies have to be performed in order to consider either permanent catheters or dialysis grafts in patients in which a short life expectancy is anticipated.

\section{Anatomical Position of Grafts}

Most grafts are placed in the forearm either in a straight shape between the radial artery and a elbow vein or in a curve shape (the so called loop graft) between the brachial artery and a elbow crease (Palder S et al: Ann Surg 1985;202:35-39).

Whenever an elbow crease fistula is preferred, when an upper arm vein seem to be adequate, most of grafts are placed at the upper arm between the brachial artery and a large vein in the lower part of the axilla (Polo et al: Artif Organs 1995;19:1181-1184). Other less frequent positions are: brachioaxillary (Polo JR et al: Am J Kidney Dis 1990;16:465-468); axilo-axillary (McCann RL: J Vasc Surg 1996;24:457-462); femoro-femoral (Korzets A et al: Nephrol Dial Transplant 1998;13:1215-1220); axillofemoral (Rueckerman et al: ANNA Journal 1991;18:567-571). In cases of caval obstruction a graft to the right atrium has been performed (El-Sabrout RA, Duncan JM et al: J Vasc Surg 1999;29:472-478). On the other hand arterio-arterial grafts in several positions have been used in cases of obstruction of 
superior and inferior vena cava (Scholz $\mathrm{H}$ et al: in Henry ML Proceedings of Vascular Access for Hemodialysis VI, Miami, Precept Press, 1999, pp 255-262).

\section{Technical Approach}

My personal choice is to perform all upper arm arteriovenous grafts, included brachiojugular prosthesis, under local anesthesia and in an ambulatory surgical setting (Polo et al: Ambulatory Surgery 1997;5:149-151). After dissection of the artery and the vein a tunneler is introduced under the skin and the graft is immediately in the subcutaneous space avoiding a long exposure of the prosthesis in the operating field. The graft vein anastomosis is first performed (the posterior wall from inside) and a clamp is placed in the graft close to the vein. The venous micro-bulldogs are released and the graft vein anastomosis is checked under a low pressure flow. Afterward, the arterial graft anastomosis is performed and, before the suture is tied, the graft clamp placed in the venous site is released allowing the blood fill the graft. The arterial anastomosis is then tied and the arterial clamps removed. In this way, blood remain flowless in the graft for a few second and heparin is not needed.

\section{Long Term Results}

I will present my experience with 716 grafts performed between 1981 and 1997. In this period the following accesses were performed in our unit: 1,259 radiocephalic arteriovenous fistulae (AVF) (67\%), 468 upper arm AVF (18\%), 82 dacron cuff catheters (3\%) and 716 PTFE grafts (28\%). Anatomical position of grafts were as follows: upper-arm (6-8 mm) 324 (45\%); upper-arm (6 mm) 184 (26\%); stright forearm $(6 \mathrm{~mm}) 96(13 \%)$; curve forearm $(6 \mathrm{~mm}) 49(7 \%)$; brachiojugular (6 and 6-8 mm) 51 (7\%); femoro-fermoral $(6-8 \mathrm{~mm}) 12$ (2\%). Overall complication rate was of 0.49 episodes per graft-year of follow-up. Complication rate (episodes per graft-year) was significantly lower in 6-8 mm upper arm grafts $(0.33)(\mathrm{p}<0.001)$, comparing with the rest of grafts $(6 \mathrm{~mm}$ upper-arm $=0.57 ; 6 \mathrm{~mm}$ straight forearm $=0.62$; brachiojugular $=0.72 ; 6 \mathrm{~mm}$ curve forearm $=0.74$ ). Overall primary patency was $70 \%, 34 \%$ and $12 \%$ at 1,5 and $10 \mathrm{yrs}$. Overall secondary patency (after successful rescue surgery for complications) was: $86 \%, 66 \%$ and $39 \%$ at 1,5 and 10 yrs. Comparison of patencies, primary and secondary was significant (log-rank, $\mathrm{p}<0.001$ ). Best patency rates were observed in 6-8 $\mathrm{mm}$ upper-arm grafts: primary, $92 \%, 52 \%, 24 \%$ at $1.5,10$ years; secondary, $96 \%$, $79 \%$ and $54 \%$ at $1,5,10$ yrs. These grafts also showed the lower complication rate $(0.33$ episodes per graft-year).

Under a policy of constructing an autologous access whenever possible, either in the wrist or the elbow, most of grafts should be placed in a upper arm position (between the brachial artery and a large vein in the distal axilla). Proximal axillary vein should be avoided in order to be able to reach a potential graf-vein stenosis under local anesthesia. Otherwise, these stenosis have to be treated by radiological angioplasty.

To get a good secondary patency, a routine program of surveillance (better with access flow measurements by means of echodoppler studies or Transonic analysis), and early treatment of thrombosis and stenosis is mandatory.
10

\section{Arteriovenous Fistula in Children}

P. Bourquelot

Clinique Jouvenet, Paris, France

First described in 1966, AVF was soon considered as the best hemodialysis angioaccess in adults. In children the first publications indicated $50 \%$ immediate failure due to small size vessels, often reduced by spasm provoked by arterial dissection. We reported our first experience of using microsurgery for AVF in children in 1978 [1] and we published our results for AVF microsurgery in children under $10 \mathrm{~kg}$ in 1981 . In 1984 the benefit of microsurgery was emphasized by a Canadian team [2] and in 1988 by an Italian one [3]. In 1990 we [4] reported 380 children receiving microsurgery for AVF: the immediate patency rate was $96 \%$ and the 24 month patency rate was $85 \%$ in distal radial-cephalic AVF, 72\% in brachial-basilic AVF, $47 \%$ in brachial-cephalic AVF and only 5\% in AV bridge-grafts.

\section{Major Technical Rules}

The condition of the vein is assessed by careful preoperative clinical examination; a venous angiography is necessary if there is any doubt, particularly in very young children. Prophylactic broad spectrum antibiotic is prescribed. The rules for suturing are: forceps must never grasp the intima, the adventitia is incised, not resected, high pressure clamps must be avoided, the thinnest possible needles are to be used. Preventive hemostasis, using a pneumatic tourniquet, makes extensive arterial dissection for clamping unnecessary: arterial spasm and ischemia are avoided.

\section{Various AVF \\ Radial to Cephalic AVF}

The cutaneous incision is longitudinal, half way between the cephalic vein and the radial artery in the wrist. The nerves must be carefully preserved. The vein is freed by a wide dissection, a ligature being placed on the collateral branches: this avoids the use of an electric scalpel that could damage the vascular trunk itself. The diameter of the vein is measured. The vein is irrigated frequently in order to prevent drying and it is only handled by its adventitia.

After section of the vein above a distal ligature, posterior incision of approximately $10 \mathrm{~mm}$ in length is made in the proximal vein. The anterior wall of the artery is exposed, without dissecting the arterial trunk. Threads are used to hold back the skin and expose the site during the anastomosis procedure. A longitudinal arteriotomy is commenced with a disposable ophthalmologic scalpel, taking great care not to damage the posterior wall. This is completed with scissors. If a few drops of blood appear they are rinsed with heparin saline and the blood is dried with small sponges. The anastomosis is made with four running sutures, by suturing first the proximal angle. It is preferable to keep the running suture untightened at the beginning so as to leave the lumen of the artery open. Frequent use of the zoom facilitates this very precise part of the operation; of course the needle must be repositioned in the needle holder for each passage through a vessel. Both proximal running sutures (posterior and anterior) are stopped at the middle of the arteriotomy. The rest of the anastomosis is made in starting from the distal angle. The tourniquet is then released. If there is a leak, an additional suture is added. Patency is confirmed by Doppler 
ultrasound, as the murmur can be absent during the first post-operative hours particularly in small children. It is very important to ascertain that the vein is not stenosed at the upper limit of the dissection.

Variations: Two running sutures anastomosis. The proximal suture is made first, starting at the middle of the posterior wall of the arteriotomy, going up to the proximal angle, and down to the middle of the anterior wall. The distal suture is made afterward. This technique gives an excellent vision on the arterial lumen.

It is often necessary to surgically transpose subcutaneously the vein two months later in small children with thick adipose tissue.

\section{Ulnar to Basilic AVF}

A sufficient length of the vein, which is situated quite far back from the artery, must be freed. It can be a help to put the forearm in flexion at the elbow during dissection. The artery is approached by passing behind the flexor carpi ulnaris. The interval before puncture is longer than with a radial to cephalic AVF.

\section{Brachial to Cephalic AVF}

A transverse incision on the anterior aspect of the elbow exposes the medial branch of the cephalic vein which must be sufficiently freed to allow it to come into proximity with the brachial artery without traction. Here again, using a pneumatic tourniquet avoids having to free the artery.

Variations: Anastomosis between the brachial artery and the cephalic vein by means of the medial branch of the basilic vein, which will be perfused in a retrograde way in the absence of valves, is preferable. Both vessels, which follow almost superimposed routes, are approached by a short antecubital incision. The vein is divided as high as possible and, after minimal dissection, its distal segment is anastomosed to the brachial artery. It is essential to ligate the perforating vein of the elbow to avoid the risk of high flow with cardiac overload. Ligation of the upper part of the forearm cephalic vein is also necessary to avoid the risk of retrograde perfusion of the forearm, which would impair venous drainage of the hand. Both these risks mean that retrograde $\mathrm{AVF}$ and side to side $\mathrm{AVF}$ at the elbow must not be used.

\section{Brachial to Basilic AVF with Subcutaneous Transposition}

The basilic vein follows a route which rapidly becomes deep above the elbow, meaning that it is often undamaged. Its use for angioaccess necessitates surgical transposition, even in the thinnest children. Its transposition is much easier when it is performed during a second stage, the first consisting of a simple AVF at the elbow which results in dilatation of the vein and thickening of its walls. The initial AVF is performed on the medial branch of the basilic vein at the elbow or, if necessary, on its lateral branch. Transposition performed two months later necessitates a longitudinal incision on the medial side of the upper arm. Collaterals of the veins are ligated and divided. Dissection downwards must be performed to the initial AVF because it is necessary not to leave a venous 'cul de sac' implanted on the artery. Tunnelization of the vein is very superficial and follows a straight line. Reimplantation on the artery is performed as low as possible. An interval of three weeks must be respected before puncturing.

\section{Femoral to Saphenous AVF, with Transposition}

This type of vascular access is very rarely used, particularly because the risk of infection is greater in the thigh than in the upper limb. Superficial transposition is either in a straight line with an anastomosis on the distal superficial femoral artery, or in a loop with an anastomosis on the common femoral artery.

\section{AV Bridge-Graft}

In this case a graft (autologous greater saphenous vein, biograft or PTFE) is implanted between an artery and a vein. It is located under the skin in such a way as to be accessible to puncture.

\section{Indications}

- In case of emergency and if peritoneal dialysis is impossible, a central venous catheter must be inserted. A subclavian catheter is to be avoided because of the risk of interference with the venous drainage of the limb with dramatic consequences for the future. An internal jugular vein catheter is preferable.

- In all other cases, children must have a distal radial to cephalic AVF, if possible, created several months ( 3 months) before use. In children under $10 \mathrm{~kg}$ the delay is longer. When distal AVF is not possible, proximal AVF is indicated: the cephalic vein is used first, then the basilic vein.

- Superficialisation of the greater saphenous vein, or even the superficial femoral vein, is exceptional and reserved for the most difficult cases, i.e. children whose venous capital of both upper limbs has been exhausted or those with bilateral proximal venous stenosis when peritoneal dialysis is ineffective or impossible.

- Bypasses, still recommended by a few authors, must be avoided at all costs in children in view of the rapidity with which they become complicated by stenosis of the venous anastomosis [5].

\section{Conclusion}

Microsurgery has greatly improved and simplified the creation of AVF in children.

\section{References}

1. Bourquelot P, Wolfeler L, Lamy L: Microsurgery for haemodialysis distal arteriovenous fistulae in children weighing less than $10 \mathrm{~kg}$. Proc Eur Dial Transplant Assoc 1981;18:537.

2. Yazbeck S, O'Regan S: Microsurgery for Brescia-Cimino fistula construction in pediatric patients. Nephron 1984;38:209.

3. Bagolan P, Spagnoli A, Ciprandi G, Picca S, Leozappa G, Nahom A, Trucchi A, Rizzoni G, Fabbrini G. A ten-year experience of BresciaCimino arteriovenous fistula in children: Technical evolution and refinements. J Vasc Surg 1998 Apr;27(4):6.

4. Bourquelot P, Cussenot O, Corbi P, Pillion G, Gagnadoux MF, Bensman A, Loirat C, Broyer M: Microsurgical creation and follow-up of arteriovenous fistulae for chronic haemodialysis in children. Pediatr Nephrol 1990; 4:156.

5. Bourquelot P, Gagnadoux MF: Vascular access for haemodialysis in children - Letter. Pediat Nephrol 1997;11:659. 
11

\section{Vascular Access in Elderly and Diabetics}

M.K. Lazarides

Department of Vascular Surgery, Demokritos University of Thrace, Alexadroupolis, Greece

The initial access of choice in ESRD (end stage renal disease) patients is the wrist radial-cephalic autologous arteriovenous fistula. However controversy exists regarding the optimal initial access site in specific subgroups of patients as the elderly and the diabetics.

Elderly: The increased interest in the elderly patients recently, is due to a tremendous demographic change within the ESRD population worldwide. It is interesting that elderly patients, who were excluded from dialysis in the early years, are now the fastest-growing segment of the total dialysis population. It has been estimated that those over 65 years of age comprise more than $60 \%$ of the total number of ESRD patients. In those patients parameters such as long-term patency and conservation of proximal access sites have been considered of minimal importance because of their limited life expectancy. Additionally many authors report inferior medium-term patency of distal autologous AV fistulae in the elderly [1, 2], while other clinical series present inverse results [3, 4]. Based on previous data proposals for more liberal use of synthetic grafts had been suggested. However dialysis techniques and supportive medical therapies continue to improve each year, resulting in better likelihood of survival of elderly ESRD patients [5]. Therefore the concept of using synthetic grafts as initial procedure in the elderly should be taken into account with skepticism. Procedures as the basilic vein transposition make an attractive alternative when the construction of a radialcephalic or brachial-cephalic autologous fistula is not feasible.

Diabetics: These patients commonly display medial calcification of the radial artery. The latter when haemodynamically significant prevents the compensatory increase in the arterial flow and therefore the maturation process in distal AV fistulae. Medial calcification known and as Moenckeberg's sclerosis is rare in brachial artery. On the basis of the previous concept some authors express little enthusiasm to create forearm AV fistulae in diabetics [6]. The construction of proximal upper-arm AV fistulae as initial approach has been suggested instead [7]. However in many diabetics a preoperative plain X-ray of the hand reveals absence of medial sclerosis and therefore the construction of a distal AV fistula is feasible. The use of the following algorithm helps in decision making.

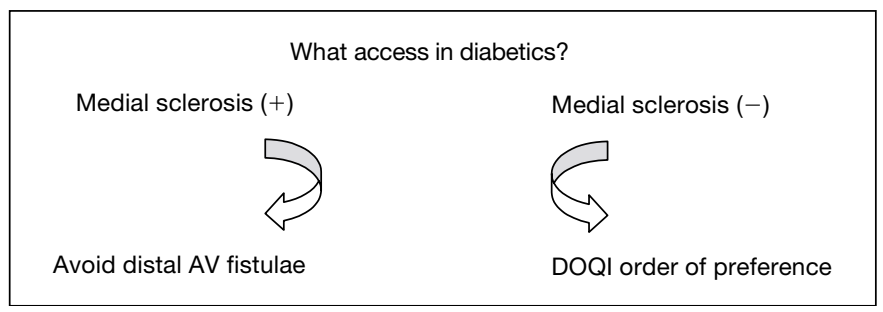

\section{References}

1. Staramos DN, Lazarides MK, Tzilalis VD et al: Patency of autologous and prosthetic arteriovenous fistulae in elderly patients. Eur J Surg 2000;166: 777-781.

2. Miller PE, Tolwani A, Lusky CP et al: Predictors of adequacy of arteriovenous fistulas in haemodialysis patients. Kidney Int 1999;56:275-280.
3. Colledge J, Smith CJ, Emery J et al: Outcome of primary radiocephalic fistula for haemodialysis. Br J Surg 1999;86:211-216.

4. Wolowczyk L, Williams AJ, Donovan KL et al: The snuffbox arteriovenous fistula for vascular access. Eur J Vasc Endovasc Surg 2000;19:70-76.

5. Peri UN, Fenves AZ, Middleton JP et al: Improving survival of octagenarian patients selected for haemodialysis. Nephrol Dial Transplant 2001;16: 2201-2206.

6. Hakaim AG, Nalbandian M, Scott T: Superior maturation and patency of primary brachiocephalic and transposed basilic vein arteriovenous fistulae in patients with diabetes. J Vasc Surg 1998;27:154-157.

7. Konner K: Primary vascular access in diabetic patients: an audit. Nephrol Dial Transplant 2000;15:1317-1325.

\section{Vascular Access Complications - Aetiology and Diagnostic}

\section{2 \\ Vascular Access and Intimal Hyperplasia \\ J.H.M. Tordoir \\ Department of Surgery, University Hospital Maastricht, The Netherlands}

The most prominent complication of hemodialysis AVF failure is uncontrolled intimal thickening by proliferation and migration of vessel smooth muscle cells, i.e. intimal hyperplasia, leading to progressive stenosis and subsequently thrombosis. This feature occurs in $20 \%$ of native AVFs and $80 \%$ of graft AVFs.

\section{Factors Influencing Intimal Hyperplasia}

\section{Blood Flow and Intimal Hyperplasia}

Intimal hyperplasia often occurs at the arteriovenous anastomosis in native AVFs and at the graft-vein anastomosis in prosthetic AVFs. Presumed to be of great importance in the development and progress of intimal hyperplasia is a certain hemodynamic factor which involves the interaction between the vessel wall and the bloodstream, namely wall shear stress (WSS).

Wall shear stress is the frictional force exerted by the circulating blood column on the endothelial monolayer covering the inner vessel wall. WSS is believed to play a significant role in the physiological adaptation of the vascular wall, in that it induces artery dilatation with increasing volume flow and vice versa. Acute changes in blood flow induce the release of vasoactive molecules by the endothelium, whereas chronic changes induce vascular remodeling. WSS is associated with the development of intimal hyperplasia. There is no conclusive data indicating whether high or low WSS is related to the development of intimal thickening. The endothelial cell plays a pivotal role in this process. Mechanisms are mechanotransduction processes that lead to biophysical, biochemical and gene regulatory responses of endothelial cells to hemodynamic shear stresses. These mechanisms include endothelialmediated alterations in coagulation (prostacyclin; NO; protein $\mathrm{C}$ and $\mathrm{S}$ ), leukocyte and monocyte migration, proliferation of smooth muscle cells (PDGF, angiotensin II, NO, TGF- $\beta$ ), and signal transduction. 


\section{Peri-anastomotic Mismatch in Elastic Properties}

In addition to WSS, peri-anastomotic compliance mismatch, i.e. mismatch in elastic properties, seems to be an important factor in the development of intimal hyperplasia.

A mismatch in elastic properties may affect intimal hyperplasia in 3 ways:

a. Mismatch leads to a point of excessive mechanical stretching, which may result in vessel wall injury and initiate a vascular response resulting in intimal thickening.

b. Cyclic stretching has a positive influence on replication of VSMC and production of extracellular matrix (ECM). In vitro studies has demonstrated that VSMC start to produce ECM and to replicate when they are subjected to high levels of distension. This phenomenon is more pronounced in VSMC derived from veins.

c. Compliance may influence flow characteristics. Flow studies have demonstrated that a sudden increase in compliance is associated with enhanced particle residence time, flow stasis and separation, suggesting low shear conditions.

\section{Treatment of Intimal Hyperplasia}

In animal experiments, pharmacological interventions, like aspirin, dipyradamole or heparin may decrease IH. Adaptation of graft geometry by banding, tapering or cuff implantation may have beneficial effects on IH in animals; however in humans no decrease in IH was shown in randomised studies. After initial enthusiasm of brachytherapy, the results in clinical studies seems not as good as expected. New preliminary data show that the immunosuppressive drug rapamycine could have an effect on IH. Rapamycine incorporated into stents has been shown to have effects in coronary arteries and the results seems to be promising.

In conclusion, intimal hyperplasia is the key event that jeopardise coronary bypass grafts, PTA, peripheral vascular grafts and hemodialysis access AVF. At present, no durable effective treatment has been developed to influence the process of IH.

\section{3}

\section{Clinical Diagnosis of Stenosis and Thrombosis}

\section{J. Pengloan}

Unité d'Hémodialyse Chronique, CHU Bretonneau, Tours, France

Hemodialysis vascular access failure represents a major source of morbidity and mortality in Chronic Hemodialysed Patients (CHD). Thrombosis is the main cause of failure of Vascular Access (VA); palpation and auscultation easily diagnose it: absence of thrill and bruit and impossibility to drawn blood through dialysis needle. The major predisposing factor of thrombotic complications is stenosis related to the development of intimal hyperplasia.

To prevent thrombosis the surveillance of VA has to identify VA at high risk for thrombosis and to determine the optimal time for correction of causative lesion. Stenosis can be located at every area in the venous outflow tract, from the Arterio-Venous Anastomosis (AVA) to the heart; however in the majority of cases, stenosis are located at the
AVA for native fistulae and at the Veno-Venous Anastomosis (VVA) for prosthesis. Consequences of stenosis are hyperpressure upstream the stenosis, hypopressure downstream the stenosis and decrease blood flow access; these signs are currently observed in graft but can be altered in native fistula because development of collateral veins. Clinical examination associated with collection of dialysis parameters should be able to detect hypopressure state and reduction of arterial blood flow (flat VA, low arterial pressure, insufficient bloodflow through dialysis pump.) and hyperpressure state (tenseness of access, elevated venous pressure (VP), prolonged bleeding after venipuncture, aneurysms). A relationship had been observed between outflow venous stenosis and venous pressure; elevated venous pressure is associated with an increased risk for thrombosis; VA surveillance protocol based on routine observation of VP (dynamic and/or static) resulted in a significant reduction of thrombosis rate but failed to eradicate thrombosis events. Dialysis parameters have limits and are not sensitive; venous pressure may detect stenosis but is a moderate predictor of access thrombosis. Non invasive measurements of VA blood flow based on dilution methods have been proposed. A close relationship has been observed between the risk for thrombosis and level of access flow and between the risk for thrombosis and decreasing of access flow between two measurements.

Graft is suspected to be at high risk for thrombosis if there are clinical signs of hyperpressure and/or elevated venous pressure (higher than $150 \mathrm{~mm} \mathrm{Hg}$ for $300 \mathrm{ml} / \mathrm{mn}$ blood flow), graft flow lower than $800 \mathrm{ml} / \mathrm{mn}$ or decreased by $25 \%$ between two measurements. Thrombosis is imminent if graft flow is lower than $600 \mathrm{ml} / \mathrm{mn}$. For Native AV fistula in-line techniques for access flow measurements are suitable only if the segment upstream the venous needle is free of collateral veins; despite this limitation monthly monitoring has proved to be effective in reduction of total thrombosis rate in native fistula. A native fistula is suspected to be at high risk for thrombosis if there are clinical signs of hypopressure, if native fistula flow is lower than $400 \mathrm{ml} / \mathrm{mn}$ or decreased by $25 \%$ between two measurements.

In-line measurements of access flow appeared to be reliable to identify accesses at high risk for thrombosis. Several devices are able to provide accurate access flow results: the Transonic System monitor (ultrasound velocity dilution) is the best-validated device, the CritLine (hematocrit dilution technique), the Diascan (ionic dialysance), and the HDM Gambro (differential conductivity technique). Some of them can be easily used at each session of dialysis.

The routine measurement of flow access is relevant to identify VA at high risk for thrombosis and is changing the management of VA.

Despite this progress some frustrating points remain:

- the occurrence of thrombosis despite intensive surveillance;

- the optimal threshold level or decreasing percentage of access flow has not been determined.

Nevertheless an optimal frequency of screening can be proposed:

- dynamic venous pressure and arterial pressure at each session of dialysis;

- clinical examination at each complain of patient or nurses;

- monthly flow measurement for graft and native fistula; Doppler flow measurement can be proposed for fistula with collateral veins. 
14

\section{Factors Predicting or Influencing Early Failure of AV Fistulae}

\section{A. Bakran \\ University of Liverpool, The Royal Liverpool University Hospital, Liverpool, UK}

It is suggested that up to $30 \%$ of radiocephalic AV fistulae fail, either due to early thrombosis or because the fistula vein does not dilate to allow haemodialysis [1]. This failure rate may be due to diseases of the radial artery itself, inadequacy of the cephalic vein, to poor surgical technique as well other host factors. The latter can only be addressed by better training and meticulous attention to detail in the performance of surgery. However, there is now sufficient evidence that preoperative duplex scanning can elucidate the morphology of artery and vein before surgery, thereby allowing problems to be picked up before surgery. Research in Liverpool has shown the duplex scanning is better at detecting diseases of vessels than clinical examination alone [2]. Disappointingly, intra operative flow measurements have not been predictive of fistula success but duplex scanning during the follow up period has. The velocity of flow measurements at $24 \mathrm{~h}$ post $\mathrm{AV}$ fistula creation has been shown to distinguish between
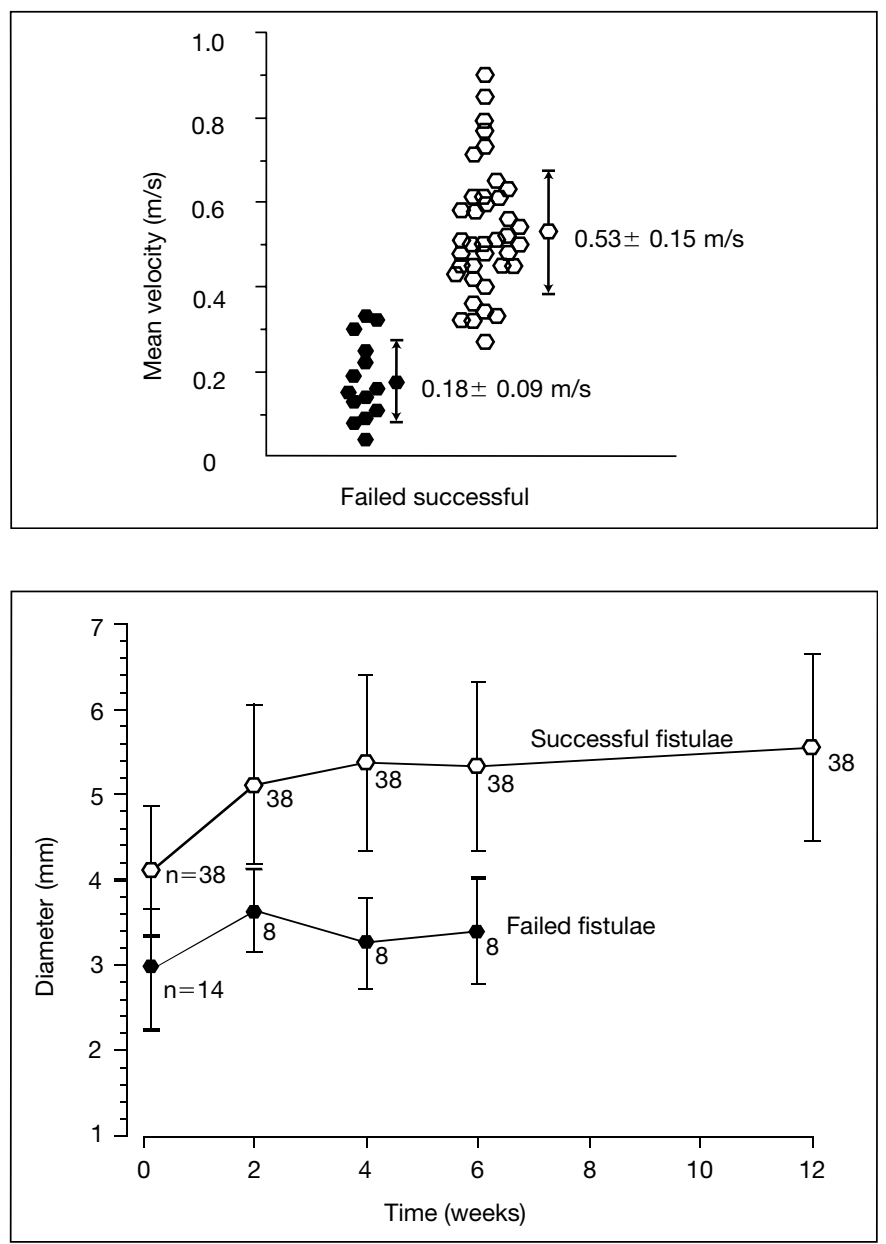

Preprint Blood Purif, Vol. 20, 2002 those fistulae which will go on to be successful and allow haemodialysis (mean velocity of flow $0.53 \mathrm{~m} / \mathrm{s}$ ) compared to those more likely to fail (mean velocity of flow $<0.2 \mathrm{~m} / \mathrm{s}$ ).

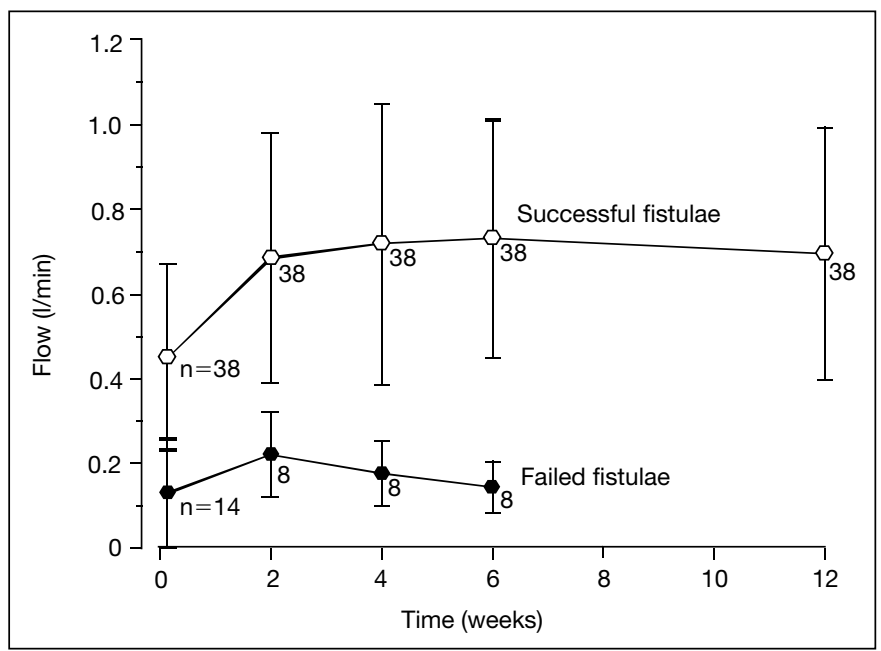

Furthermore, duplex scanning of AV fistula over the subsequent few weeks to assess fistula blood flow and vein diameter has been shown to separate the successful and failing fistula pattern.

Therefore, measurements of velocity of flow at $24 \mathrm{~h}$ and fistula flow at two/three weeks post-operative should provide good predictions of success or failure of AV fistulae. This allows surgeons to either revise the unsuccessful fistula, or to create another AV fistula at a different site. No longer need a surgeon prevaricate in his decision making when reviewing patients with radiocephalic AV fistula problems following surgery.

\section{References}

1. Palder SV, Kirkman RL, Whittlemore AD, Hakim RM, Lazarus TM, Tilney NL: Vascular Access for Haemodialysis: Patency Rates and Results of Revision. Ann Surg 1985;202:235-239.

2. Wong V, Ward R, Taylor J, Selvakumar S, How TV, Bakran A: Factors Associated with Early Failure of Arteriovenous Fistulae for Haemodialysis Access. Eur J Vasc Endovasc Surg 1996;12:207-213.

15

\section{High Blood Flow Reduction}

\section{P. Bourquelot \\ Clinique Jouvenet, Paris, France}

\section{Introduction}

Although a blood flow rate of 300 to $500 \mathrm{ml} / \mathrm{min}$ is sufficient to ensure haemodialysis in adult patients, the actual AVF flow is usually much higher [1]. Apart from proceeding as distal as possible, there is no reliable way to limit blood flow when creating an AVF.

The magnitude of AVF blood flow depends of:

1. The initial diameter of the artery, while the role of the initial calibre of the arteriovenous anastomosis remains controversial. As 
known from congenital AVF, the more proximal is the AVF, the earlier and important is the cardiac overload.

2. The ability of the artery to dilate with time. Among therapeutic AVF, the distal cephalic-to-radial AVF, which has the longest life, is frequently involved in high blood flow.

High flow appears infrequently in two kinds of angioaccesses:

1. Distal basilic-to-ulnar AVF, probably due to the small diameter of the ulnar artery.

2. Prosthetic arteriovenous bridge-graft, probably because of the early venous anastomosis stenosis.

A flow reduction is necessary when flow, related to the body surface area of the patient, is important and/or when a cardiac overload is observed. In cases of associated distal ischemia, flow reduction to decrease vascular steal must be considered as well as the treatment of any artery stenosis, the revascularisation of distal arteries and the ligation of the AVF.

\section{Closure of the Arteriovenous Angio-Access}

After a successful kidney transplantation or when it is possible to move to peritoneal dialysis, the closure of the permanent arteriovenous fistula can be done.

At the forearm, ligation of the vein near the anastomosis is possible; in case of anastomotic aneurysm, it may be necessary to interrupt the artery. At the elbow, the brachial artery must be spared or patched. In both cases, one must not forget the risk of postoperative hypertension, especially in children.

In a few cases, it is possible to close a proximal AVF and to replace it by a more distal permanent access on either of both upper limbs. Obviously in case of multiple fistulas, the ligation technique is also indicated.

The popular elbow side-to-side and the perforating vein arteriovenous fistulas deserve many criticisms: retrograde flow to the forearm may induce painful and ischemic distal venous hypertension; perfusion of the profunda brachial veins via the perforating vein will not give any chance of percutaneous venipunctures; the same remark applies to the basilic vein which is not to be punctured without an important risk of brachial artery damages, unless previous surgical superficialisation. Arterial distal ischemia, very frequent in diabetics ( $25 \%$ for J. Elfström) [2], and cardiac overload are the major unrecognised risks of this technique (H. Pizakatzer) [3]. Closure of the anastomosis straight above the artery is to be done and a new side to end 'only one downstream cephalic or superficialised basilic vein' anastomosis is performed.

\section{Banding}

Banding reduction of the venous diameter was the method of choice, but this procedure rarely results in actual flow reduction.

Objections to banding are multiple:

1. A peroperative flowmetry is required but prior surgical dissection induces spasm and inaccurate measuring of the flow.

2. Reduction of vascular calibre must be important to avoid the risk of persistent high flow and precise to avoid the risk of thrombosis. Such venous constriction has sometimes been replaced by a constrictive perivenous mesh prosthesis or by the interposition of a short stepped (4 to $6 \mathrm{~mm}$ ) PTFE graft. However the degree of reduction in calibre used in both cases is insufficient and no quantitative flow results have been published.

\section{Arterio-Arterial Bypass}

In the seventies we gave up early the project to change high flow AVF into arterio-arterial bypass. The theoretical advantage was a marked reduction of basic blood flow, capable to increase highly after cannulation for haemodialysis. The 10 artery-to-artery bypasses which we had created were complicated with early thrombosis, distal artery embolism and painful dialysis due to high-pressure arterial reinjection.

\section{Proximal Artery Ligation}

In an end-to-side fistula at the forearm, the major part of the inflow is supplied by the proximal artery, and the remaining comes from retrograde flow through the palmar arch and the distal artery. Ligation of the distal artery was performed by Anderson [4] first for treatment of steal syndrome. We routinely use ligation of the proximal artery [5] for treatment of high blood flow. The surgical procedure is very simple and a $50 \%$ reduction in flow through the AVF can be expected.

This method also applies in some cases of proximal high flow AVF coexisting with high bifurcation of the brachial artery which seems to be present in $14 \%$ of individuals. High bifurcation diagnosis is made on the existence of an 'arteriovenous' Doppler signal on both distal forearm arteries (retrograde in one). The AVF, although situated at the elbow, actually involves radial or ulnar artery which, if no angiographic arterial stenosis is present, can be ligated proximally without risk of distal ischaemia.

After proximal artery ligation at the wrist, high flow has persisted or reappeared postoperatively in a few cases. Associated ligation of the distal artery, reducing AVF in-flow to small branches from ulnar and interosseous arteries, may be necessary with a $34 \%$ mean reduction rate.

\section{Distal Artery Extension}

Distal artery extension involves replacing inflow from brachial artery of a proximal AVF by inflow from one of its smaller calibre distal branches. The original arteriovenous anastomosis is closed at the elbow; an arteriovenous bridge graft is connected between the distal radial or ulnar artery at the forearm and the out-flow tract (vein or rarely prosthesis) at the elbow. A $50 \%$ mean reduction rate is observed. In a few cases, this method may be impossible, due to diabetic distal artery calcifications. Its draw back is eventual future occurrence of a bridge-graft venous anastomosis stenosis.

\section{Radial Artery Transposition}

Radial artery transposition, less frequently used, is also a surgical procedure to reduce excessive blood flow through a proximal arteriovenous fistula: the brachial artery which supplies the vein is replaced by the radial artery which calibre is much smaller. For that purpose, the radial artery is first dissected from the forearm, divided at the wrist and then turned upwards to reach the vein at the elbow. Mean reduction rate is $65 \%$.

No prosthesis, with its risk of venous anastomosis stenosis, is used. This method seems useful in young patients with normal forearm arteries, specially in children in which bridge-grafts stenosis appears early while long term angioaccess is necessary.

\section{References}

1. Levy BI, Bourquelot P, Ponsin JC, Man NK, Martineaud JP: Noninvasive and invasive blood flowmetry in hemodialyzed patients with high blood flow fistulas. Trans Am Soc Artif Intern Organs 1984;30:335-337. 
2. Elfstrom J, Lindell A: Limitations of the use of arteriovenous fistulae in the cubital fossa. Scand J Urol Nephrol 1994;28:123-126.

3. Piza-Katzer H, Laszloffy P, Schidrich R: Complications of antecubital arteriovenous fistula. Vasa 1994;23:163-166.

4. Anderson CB. EEE, Harter HR, Graff RJ, Codd JE: Local blood flow characteristics of arteriovenous fistulas in the forearm for dialysis. Surg Gynecol Obstet 1977;144:531-533.

5. Bourquelot P, CP, Cussenot O: Surgical improvement of high-flow arteriovenous fistulas, in Sommer B, Henry ML (eds). Vascular access for hemodialysis, Pluribus Press, USA 1989;124-130.

\section{6}

\section{Access Flow Surveillance: Methodology and Accuracy in Predicting Stenosis, Thrombosis, and Intervention Effectiveness}

\section{N.M. Krivitski}

Transonic Systems, Inc., Ithaca, NY, USA

Over the last five years, more than 200 publications have been focused on the development and clinical implementation of access flow (AF) measurements during hemodialysis. Ultrasound dilution technology utilizing the reversed hemodialysis line position has appeared to be the most widely used. To increase the accuracy of the technology, the following aspects were addressed during development:

1. Complete mixing of indicator with access blood. To ensure complete indicator mixing, hemodialysis lines are reversed, and the indicator (saline) is introduced into the venous line. The mixture of access blood and indicator is withdrawn into the arterial line where a dilution curve is detected by an ultrasound dilution sensor (or different sensor type for other technologies). It has been shown that a pump flow in the range of 200 to $300 \mathrm{ml} / \mathrm{min}$ allows for complete mixing to take place for access flows up to $4,000 \mathrm{ml} / \mathrm{min}$.

2. Elimination of the influence of cardiopulmonary recirculation $(C P R)$. With the reversed line position, indicator that returns to the arterial sensor site through CPR falsely increases the area under the dilution curve. This compromises AF accuracy mostly in the average to high ranges ( 1,000 to $4,000 \mathrm{ml} / \mathrm{min})$. A special algorithm is used to separate out the CPR component to obtain accurate measurements for the whole AF range.

3. Elimination of operator dependence. The placement of an additional sensor on the venous line detects the amount of introduced indicator. In the calculation of AF, only the ratio of introduced (venous line) and recorded (arterial line) indicator is used. This eliminates the influence of injection volume and time duration of injection on $\mathrm{AF}$ measurement results.

4. Elimination of the influence of injection flow. For AF measurements, 10 to $25 \mathrm{ml}$ of saline is introduced into the vascular access. To eliminate the influence of this injection on $\mathrm{AF}$, the indicator is specifically introduced at a site far from the vascular access (arterial line for saline release $20-25 \mathrm{ml}$, venous bubble trap for $10 \mathrm{ml}$ saline injection) to separate the introduced volume change from the dilution curve.

More than 43 publications have been focused on evaluating the use of access flow to predict thrombosis and hemodynamically significant stenosis $>50 \%$. Thresholds (analogous to K/DOQI Guidelines) of low AF ranged from $500-800 \mathrm{ml} / \mathrm{min}$ with $600 \mathrm{ml} / \mathrm{min}$ being the most commonly used. The use of monthly AF decreases of $20-25 \%$ as a predictor was also studied. AF measurements before and after percutaneous transluminal angioplasty (PTA) were used to evaluate intervention effectiveness.

Of these publications, 28 were further analyzed. Statistical indices of accuracy were compiled, and weighted averages (Wt Ave) were calculated (not discriminating for AF threshold).

The data suggest that AF measurements supply high accuracy in predicting access stenosis and thrombosis, and that implementing an AF surveillance program followed by PTA dramatically decreases the incidence of thrombosis events in the hemodialysis patient population.

17

\section{Aneurysms and Infection}

\author{
J. Barbosa*, F.M. José** \\ *Hospital Santa Maria, Lisboa, Portugal \\ **Hospital Garcia De Orta, Almada, Portugal
}

\section{Pseudoaneurysms}

Pseudoaneurysms of hemodialysis access are defined as a permanent, localised enlargement of a fistula, graft or of the anastomoses.

The denomination of pseudo or false aneurysm arises from the fact that the vessel or graft wall is disrupted. Pseudoaneurysms of the graft are usually related with repeated puncture at the same place or with traumatic puncture and subsequent perigraft hematoma formation.

Table to abstract 16

\begin{tabular}{|c|c|c|c|c|}
\hline Category & Access type & Pub. & Wt Average (Range) & $\mathrm{n}$ \\
\hline $\mathrm{AF}$ to predict $>50 \%$ stenosis & AVG \& AVF & 11 & PPV 84\% (56-100\%) & 316 \\
\hline AF to predict $>50 \%$ stenosis & AVF & 3 & PPV 90\% (82-100\%) & 82 \\
\hline $\mathrm{AF}$ to predict $>50 \%$ stenosis & AVG & 5 & PPV $89 \%(74-100 \%)$ & 115 \\
\hline AF to predict thrombosis & AVG \& AVF & 8 & Sens $82 \% *(70-89 \%)$ & 1,867 \\
\hline $\mathrm{AF}$ to predict thrombosis & AVG \& AVF & 5 & Spec $80 \%(72-91 \%)$ & 507 \\
\hline $\mathrm{AF}$ to predict thrombosis & AVG \& AVF & 4 & PPV 74\% (67-79\%) & 431 \\
\hline Increase in $\mathrm{AF}$ after PTA & AVG \& AVF & 9 & $250 \mathrm{ml} / \mathrm{min}(174-397 \mathrm{ml} / \mathrm{min})$ & 404 \\
\hline Decrease in thrombosis rate & AVG \& AVF & 9 & 2.3 fold $(1.3-5.4$ fold $)$ & 1,481 \\
\hline
\end{tabular}

$\mathrm{Pub}=$ number of publications; $\mathrm{n}=$ number of accesses; Sens $=$ Sensitivity; Spec $=$ Specificity; PPV $=$ Positive Predictive Value $* *=$ includes unpublished data; $\mathrm{AVG}=$ arteriovenous graft; $\mathrm{AVF}=$ arteriovenous fistula. 
This hematoma develops beneath a fibrous scarring tissue. With the continuous pressure of the arterial flow, the hematoma expands and a fibrous capsule forms.

This was well demonstrated by Delorme et al., that studied structurally and histopathologically 52 excised PTFE grafts, after being used for hemodialysis access. These exams shown that repeated needle puncture may result in a perigraft fibrous tissue capsule directly above areas where the graft was punctured. The delicate microporous structure of the graft wall was shown to be disrupted by needle punctures filled by surrounding connective tissue [6]. Anastomotic pseudoaneurysms of arterio-venous fistulas or grafts tend to develop more often in association with infection, and its physiopathological mechanism is related with the disruption of the suture line and formation of an hematoma, also contained in a fibrous capsule.

This physiopathological situation is present in 2 to $10 \%$ of the access Palder et al. [7] followed 163 PTFE grafts for a period of 4 years and found an incidence of $2 \%$. They also demonstrated that this complication is a phenomena relatively isolated in the life of each graft, with a rate of 1.3 [8].

\section{Diagnosis}

The pseudoaneurysms presents as a localised dilatation of the arterio-venous access, pulsating and expandable. The patient can refer pain over the tumafaction, as well as complains of obvious cosmetic deformity.

Sometimes, the skin over the graft is thin and damaged, and erosion and necrosis areas can develop, associated to repeated puncture. Difficulty in the sealing of needle puncture, with prolonged hemorraghe can be seen and this preceeds spontaneous bleeding and rupture of the graft. Skin erosion can also predispose to infection of the prostheses, because of its exposure. Dialysis personnel must be taught that erosions in the skin overliyng a conduit or any abnormal bleeding must be brought to the attention of a vascular surgeon.

Thrombosis is also more frequent in the presence of a false aneurysm and is related with the stagnation of blood and progressive layering of clot in the lumen of the pseudoaneurysm [9].

The diagnosis of this situation can be confirm by Eco-Doppler. This exam can define the exact nature and extension of the aneurysm and permits its differential diagnosis from hematomas, seroma, flegmon, lymphocele or abscess. It appears as a liquid formation, pulsated and the doppler analysis show arterial flow. Thrombus inside can be seen as echogenic material.

Angiography offers very good definition of the entire arteriovenous access. This information is essential for surgical correction, but its indications are limited nowadays, because Eco-Doppler permits the obtention of the necessary data. So, this exam is reserved for selected patients, in which the ultrasonographic observation by a skilled operator could not offer the complete information for surgical correction. This situation is more frequent in cases of anastomotic aneurysm or when stenoses of the drainage vessels are suspected. Venous obstruction from stenosis is present in many of these patients, and that potentiates the false aneurysm, because it tend to rise the pressure in the graft $[10,11]$.

\section{Treatment}

Surgical correction of graft pseudoaneurysms is recommend because of the high incidence of subsequent thrombosis, infection or rupture. Surgical correction in the fistulas is indicated if symptomatic (painful, infected or covered with ulcerated skin) or cosmetically unacceptable.

Various are the techniques described to treat this condition and the choice of each one depends on multiple variables like the type of access (AV fistula or graft), extension of the lesion, presence of infection, thrombosis, rupture or the aspect of the skin overlying the access.

The main objective of this surgery is to treat the false aneurysm, preventing its complications, but preserving the function of the vascular access, whenever possible.

False aneurysm of patent arterio-venous fistulas can be treated with ligation and drainage or removal of the dilatation, followed by an interposition of a prosthetic segment. In distal fistulas other option can be used, which is technically easier and avoids the use of synthetic material. It consists in the ligation with drainage or removal of the pseudoaneurysm and creation of a more proximal fistula. In long term fistulas with a very deteriorated vein, with stenoses, skin necrosis or infection, ligation and drainage can be the only possible solution. Access in other site can be created at the same time.

In degenerative conduit problems limited to a portion of the graft, the salvage can be achieved by an interposition of a segment of prostheses in situ or bypassing the lesion. The old graft can be left in place if it is not infected or exposed. This technique offers the advantage of retaining part of the original access to puncture providing dialysis continuity [12].

Other method of treatment is the ligation/exclusion of the aneurysm, with drainage or excision, without any reconstruction. This alternative is selected when the reconstruction is not technically feasible, in cases of extensive skin lesion or in emergency situations.

Infected grafts should be removed and the arteriotomy is closed with a vein patch. If the access is thrombosed, the surgery resumes to, the drainage of the thrombus or excision of the false aneurysm. These procedures are indicated in cases of severe pain or cosmetical deformity.

Emergency surgery should be performed in cases of rupture that can be as serious as exsanguinating hemorrhage. In this situation, infection must be carefully excluded because it is frequently associated with rupture and conditionates the surgical proceeding.

In the absence of evident signs of infection and whenever the hemodynamic and metabolic condition of the patient permits, reconstructive surgery, with preservation of the access function, should be attented.

If signs of infection are present, removal of the entire prostheses is mandatory. A course of i.v. antibiotics are necessary during surgical treatment of an exposed graft.

\section{Prevention}

Prevention is based in the careful needle insertion technique, with variation of puncture site correct hemostasis and the use of rigorous aseptic technique.

Needle insertion into the area of pseudoaneurysm should be avoided and any skin lesion over the conduit should be investigated and treated.

\section{References}

1. Feldman HI, Kobrin S, Wasserstein A: Hemodialysis vascular access morbidity. J Am Soc Nephrol 1996;7:523-535.

2. Back M, White R: The biologic response of prosthetic dialysis grafts. Vascular Access Principles (Mosby) 1996;3:137-149.

3. Kaufman J: Failing and failed hemodialysis access sites. Advances in Vascular Surgery (Mosby) 1997;5:61-82. 
4. Rutherford RB: The value of non invasive testing before and after hemodialysis access in the prevention and management of complications. Semin Vase Surg 1997;10:157-161.

5. DOQI: Clinical practice guidelines for vascular access. V Management of complications Optimal approaches for treating complications. Am J Kidney Dis 1997;30(suppl 3):S27-S28.

6. Delorme JM: Vascular Access for hemodialysis: pathologic features of surgical excised PTFE grafts. Am Vase Surg 1992;6:517-524.

7. Connel TP, Wilson SE, Vascular Access Surgery, 4th Edition Sauders Company, 1995;2:1233-1243.

8. Palder SB, Kirkman RL, Whitemore AD, Hakin RM, Lazarus JM: Vascular Access for Hemodialysis. Patency rates and results of revision. Ann Surg 1985;202:235-239.

9. Butler CE, Tilney NL: Hemodialysis access - Permanent; in Jacobs C, Kfellstrand CM, Koch KM and Wuichester JF (eds): Replacement of Renal Function by Dialysis. 1996;(4th edition):293-304.

10. Raju S: PTFE grafts for hemodialysis access. Techniques for Insertion and Management of Complications. Ann Surg 1997;206:666-673.

11. Veith FJ, Wilson SE, Hobson RW et al: Vascular Access Complications and new methods. Trans Am Soc Artif Intem Organs 1982;XXVIII: 647-650.

12. Corry RJ, Patel NP, West JC: Surgical Management of Complications of Vascular Access for Hemodialysis. Surg Gynec Obstet 1980;151:49-54.

18

\section{Infections of Vascular Accesses}

\author{
J. Barbosa*, F.M. José** \\ *Hospital Santa Maria, Lisboa, Portugal \\ **Hospital Garcia De Orta, Almada, Portugal
}

Blood stream infections in dialytic patients are common and often result in significant morbidity and mortality. They constitute the second cause of death of the dialytic patient, accounting for 6 to $18 \%$ of all deaths [1]. Moreover, the infection of the vascular access is the principal cause of sepsis in this population of patients and the second cause of loss of the access.

Infection of vascular access is common. The combine risk of infection for all type of access was of $10 \%$ in the first year. This risk is fivefold greater for the PTFE material when compared with the native vein. The rate of infection described for the prosthetic grafts was of 11 to $35 \%$, while for the native fistula of only $3 \%[2,3]$.

In the DOQI document, the combine infection rates for permanent access, including local and bacteriemic infection were of 1 to $4 \%$ for the primary $\mathrm{AV}$ fistula and 11 to $20 \%$ for the $\mathrm{AV}$ grafts.

The consequences of the infection of a vascular access are, not only its lost, but also local and systemic life threatening complications. The most dangerous local complication is the rupture of the access, accompanied by haemorrhage. They may also lead to sepsis or metastatic infections involving the spine, joints, brain and other internal organs. A small percentage of the patients can develop infectious endocarditis.

The contamination of a vascular access usually begins through the skin above it, via the hands of the personnel, medical devices or direct contact with organisms of the hospital environment. This barrier is break in each dialysis session, exposing the access to infection, even when rigorous aseptic technique is used. Infection of the graft is usually related with the needle puncture, especially if a hematoma is formed, which provides a good medium for bacterial growth.

Dialytic patients are prone to infection because of their age, renal failure and associated diseases, such as diabetes, and they do not fight infections well. The uraemic state alters the properties of the granulocites, specially the opsonization and quimiotaxy capacities, and also reduces the number of killer cells and monotic and cell-mediated T-cell activity.

The disproteinemia, anaemia and malnutrition seen in these patients can also contribute to the low resistance to infection verified in these patients. In addition, increased risk can be related with iron overload, more significant when the serum ferritin is higher than $500 \mathrm{ng} / \mathrm{l}$.

Some bacteremias are due to contamination of the dialysis equipment itself and outbreaks of hemodialysis-related bloodstream infections have been described due to dialysate contaminated with bacteria and to inadequate reprocessing of hemodialysers [4].

The hemodialysis technique itself can cause a reduction of polymorphonuclear and complement activation with the higher peak during the initial 15 to 30 minutes of treatment. It can provoke, as well, a reduction of nearly $50 \%$ of the tissue oxygen tension, and the combination of these alterations causes a greater susceptibly to lesion and infection [5].

The skin of the dialytic patients is frequently colonised with bacteria, sometimes antibiotic-resistant, that are prevalent in the dialysis units, and the acquisition of those pathogens occurs most frequently via the hands of the personnel. One important factor related with the skin contamination is the nasal carriage of Staphylococcus aureus. Several studies have demonstrated that the nasal carriage of $S$. aureus occurs in the majority of the dialytic patients, accounting for rates of colonisation as high as $75 \%[6,7]$. Studies of Ena et al demonstrated that asymptomatic nasal carriage of this specimen is predictive of the risk of developing infection of the access. This author reported that 11 of 12 staphylococcus infections were due to the same phage types and plasmid DNA endonuclease digestion patterns as prior obtained from surveillance cultures [8]. Furthermore, the risk of staphylococcus infection in the patients with nasal carriage of the bacteria can be reduced by measures to eradicate it, like the nasal instillation of topical antibiotic mupirocin. Other studies demonstrated that the actual benefit of such therapy might be due, in part, to the eradication of simultaneous hand carriage of $S$. aureus of the patients treated with intranasal therapy [9]. In spite of this, the eradication of the nasal carriage of the $S$. aureus is not consistently applied by most of the nephrologist because of its elevated cost, the necessity of special cultures to identify the carriers and the possibility of recolonization. To avoid that, antibiotic therapy has to be given in periodic intervals, with the risk of selecting methicilinresistant specimens. Furthermore, eradicating nasal colonisation with $S$. aureus in dialysis patients can result in the appearance of other difficult to treat organisms, like Pseudomonas aeruginosa [10].

Predisposing factors to infection, related with the access itself include the type of access, its location and the number of invasive revisions. Prosthetic graft are more prone to infection than native vein as we already said, and grafts located in the groin present a higher incidence of infection than access in the arm and forearm, respectively. The number of revisions is directly associated with infection.

In a recent study of 1,455 patients receiving chronic hemodialysis, followed for more than six months, a multivariate analysis of risk factors for infection selected three that were independently associated with its occurrence, including previous history of infection, the use of catheter and an elevated serum ferritin level [11].

In other study, also designed to determine which epidemiological factors were associated with permanent access infection in a population of chronic hemodialysis patients selected the type of graft and multiple PTFE graft surgical revisions as independent risk factors. The group of factors studied include also location other than the 
forearm, limited ambulatory status, residence in a nursing room, bacterial infection in a distant site and number of hospitalisations [12].

\section{Clinical Presentation}

The patient often presents with non-specific symptoms, but any delay in treatment can lead to life threatening sepsis and/or haemorrhage and also makes the salvage of vascular access impossible.

The first signs of infection are subtle, with discrete redness, swelling and pain overlying the graft. These signs can be localised to a region of the graft or extending through all the graft. In some cases, localised abscess develops and a non-pulsate tumefaccion, with flotation, redness and tenderness is observed. Abscess can rupture with suppuration, causing skin necrosis and its disruption exposes the prosthesis

False aneurysm is other complication of infection that involves the suture line of the anastomosis, and manifest as a pulsate tumefacion with inflammatory signs.

The false aneurysm and the exposion of the graft indicate dangerous situations because they preclude rupture that can be accompanied by more or less profuse haemorrhage.

General symptoms like fever and anorexia can be the initial signs of infection and should alert for it. In the dialytic patient with fever and in the absence of other symptoms all grafts should be investigated, even the occluded ones, because they can be the source of infection [13]. Fever in the presence of an infected vascular access can indicate associated sepsis, the possibility of metastatic infections of the bone, brain, and liver or bacterial endocarditis [14]. Methastatic infections can include osteomyelitis, septic arthritis, septic pulmonary embolism, liver abscess and endopthalmitis [15].

Infection of the recently implanted grafts are more difficult to detect because the inflammatory signs like oedema, redness, pain and heat, associated with some haematoma are common after this type of surgical procedures.

\section{Diagnosis}

Useful criteria for diagnosing vascular access infection have been synthesised by Fong in 1992 and include:

1. Bacteriemia associated with local inflammatory signs, without any other obvious infection.

2. Evidence of local infection with positive culture of the pus or direct aspiration.

Some tests have proved to be useful when an infection is suspected, like the Indium scans, utilized to detect infection of clotted, non-functioning grafts [13].

The Ecodoppler can allow the diagnosis of infection and its extension, if liquid surrounding the graft can be seen in association with clinical signs of infection. This exam can not exclude the existence of infection but can be useful to therapeutic decision because it permits to determine if all the graft is involved in the process.

\section{Microbiology}

Gram positive organism continues to be the most common bacteria involved in hemodialysis access infections. In fact, Staphylococcus aureus was positive in $71 \%$ of the cultures and Staphylococcus epidermitis in $10 \%$. Gram negative organisms remain a minor contributor to fistula infection [16]. Other involve micro-organisms include Streptococcus viridans, Enterococus, Pseudomonas aeruginosa, Mycobacterium and Candida albicans. In most of the cases, the infection is monobacterian, which accounts for $80 \%$ of the cases. In one third of the situations the specimen couldn't be isolated, possibly due to previous antibiotherapy.

Although Staphylococcus aureus methicilin-sensitive has remained the predominant gram-positive pathogen during the last two decades, the percentage of $S$. aureus that are resistant to methicilin has steadily increased.

\section{Treatment}

If a vascular access infection is suspected because of the presence of some local inflammatory signs or fever, empirical antibiotic therapy should be initiated, based on the specimens usually isolated in the dialysis unit. Staphylococcus aureus is the most frequent microorganism responsible for these infections, so is justified to choose a third generation cephalosporin associated to gentamicin or netromycin.

Vancomycin is a powerful antibiotic, which covers all Staphylococcus with the advantage of the commodity of administration in dialytic patients but should be kept in reserve in order to avoid the emergence of antibiotic resistance.

Cultures of blood from the access and peripheral vein should be done and the antibiotic should be changed if the antibiogram suggests so.

If inflammatory signs persist or an abscess forms near an arteriovenous graft, surgical exploration with drainage is indicate, permitting also to obtain material for bacteriological exam. The incorporation of the prosthesis in the surrounding tissues makes difficult its isolation and constitutes a sign of absence or low aggressive infection. In these cases, one can decide not to remove the entire graft and prefer more conservative options. That includes the coverage of the graft with muscle flaps or resection of the non incorporated segment of prosthesis with reconstruction by interposition of a new one, passing through a different tunnel. The segment of prosthesis removed should be cultured to determine the type of bacteria and its sensitivity to antibiotic [17, 18]. Aggressive antibiotic therapy is instituted and careful observation has to be made to exclude persistent infection.

From the point of view of a vascular surgeon, in the presence of infection, all prosthesis should be removed, with direct suture of the vein and arterioraphy with a vein patch. However, the necessity to maintain the regular dialysis program and to avoid wasting anatomic segments for vascular access justified sometimes the partial removal of the infected prosthesis, in spite of the risk of rupture of the new anastomosis, persistent infection and sepsis. In these cases, it is fundamental a close clinical and bacteriological follow-up. That is particularly important in difficult patients, which don't have many options for implantation of a new access.

However, the total removal of the graft is mandatory in the following situations: occluded and infected grafts; infections caused by very aggressive bacteria like Pseudomonas or fungus; patients that do not respond to antibiotic therapy; infection that extends through all the graft; infected false aneurysms and recurrent infection.

The removal of all the prosthesis including the segment near the anastomosis is difficult in same cases because it can be surrounded by fibrosis and very well incorporated. In those cases, to avoid vascular and neurologic lesions during the surgical dissection of the vascular elements, some studies have demonstrated a relative safety of leaving a small patch of prosthesis to close the arteriotomy and venotomy, without leading to its stenosis [19].

Other therapeutic options can be employed in order to maintain a vascular access and include the total removal of the graft associated with the anastomosis of the artery to the arterialized vein. This is 
possible especially in the cases of forearm grafts made to superficial veins and in the absence of great conspurcation. This technique of immediate reconstruction allows the surgeon, when that is possible, to use the arterialized outflows vein and preserve other sites for future use [20].

Infection of a newly placed graft (within a month) should be treated with antibiotics and remotion of the entire graft, regardless the extension of infection, because the prosthesis is not yet incorporated in the surrounding tissues allowing and its easy propagation [21].

Infections of primary arteriovenous fistula should be treated with antibiotics and all suppuration collections should be surgically drained. Closure of a fistula is necessary in cases of septic emboli or menace of rupture and in the presence of skin necrosis or false aneurysm. In that cases, a proximal arteriovenous fistula can be created using the arterialized vein in a place not infected.

\section{Prevention}

The prevention of access fistula starts with the preparation of the patient for surgery and during the implantation of the prosthesis. Any septic point should be diagnosed and treated and malnutrition, anaemia and disproteinemia corrected as much as possible, trying to optimised the general status and the resistance to infection. Coagulation defects should also be corrected in order to avoid hematoma formation and unnecessary bleeding.

The skin should be washed before the surgery and disinfected properly and strict aseptic technique should always be applied. New alternatives like riphampicin impregnated prosthesis or homografts can be use, whenever necessary, in cases of recurrent infections or immunosupression.

Antibiotic prophilaxy for the surgical procedure is usually used and a third generation cephalosporin is generally chosen, based on the fact that the Staphylococcus aureus or the Streptococcus are the most frequent infective bacteria related with the surgical implantation of the prosthesis. Some studies have advised the use of a single dose of Vancomycin before the surgery [21], but this policy can create resistance to this antibiotic, selecting resistant microorganisms and should therefore be reserved for the problematic cases.

The manipulation of a vascular access constitutes other major aspect because every time it is punctured can be contaminated. The patients is taught to wash is arm and hands before dialysis and their skin is disinfected with alcohol at $70^{\circ}$ or Iodopovidona at $10 \%$. The puncture and homeostasis technical aspects should be respected to avoid hematomas that can more easily infect.

Other controversial aspect is the timing to implant a prosthetic graft after an access infection episode. A new permanent access should not be implanted until blood cultures, performed after cessation of the antibiotic treatment, have been negative for at least 48 hours. The risk of a second infection is higher (12\%/year) because this rules can not always be respected. This is caused by the necessity to maintain the dialysis program in the presence of shortage of locals for temporary catheter implantation.

Standardised surveillance and data collection system should be implanted in each dialysis facilities in order to allow reliable data comparison and benchmarking. This program can detect outbreaks of infection that alert for the problems in the standard precautions permitting its correction. It also allows us to know which are the most frequent infective micro-organism and its sensibility to antibiotics [22].

In conclusion, the vascular access infection remains a very controversial matter and there are still no guidelines about many related issues. Therefore, the decision is frequently individual and we hope that, in the future, many other consensus can be obtained.

\section{References}

1. Cheesbrough et al: A prospective study of the mechanisms of infection associated with hemodialysis catheters. J Infect Dis 1986;154:579-589.

2. Kessler et al: Bacteriemia in patients on chronic hemodialyis. A multicentre prospective survey. Nephron 1993;64:95-100.

3. Keane et al: Incidence and type of infections occurring in 445 chronic hemodialysis patients. Trans Am Soc Artif Inter Organs 1977;23:41-46.

4. Outbreaks of gram-negative bacterial bloodstream infections traced the probable contamination of hemodialysis machines MMWR Morb Mortal Wkly Rep 1998;47(3):55-59.

5. Jensen et al: Subcutaneous tissue oxygen tension falls during hemodialysis. Surgery 1987;101:416-421.

6. Kirmani et al: Staphylococcus nasal carriage rate in patients receiving long term hemodialysis. Arch Int Med 1978;138:1657-1659.

7. Yu et al: Staphylococcus aureus nasal carriage and infection in patients on hemodialysis. N Engl J Med 1986;315:91-96.

8. Boelaert et al: Nasal and cutaneous carriage of Staphylococcus aureus in hemodialysis patients- the effect of nasal muporicin. Infect Control Hosp Epidemiol 1996;17:809-811.

9. Ena et al: Epidemiology of Staphylococcus aureus infections in patients on hemodialysis. Infect Control Hosp Epidemiol 1995;15:78-81.

10. Light et al: Ecological relation between Staphylococcus aureus and Pseudomonas in a nursery population. N Engl J Med 1968;278:1243-1247.

11. Hoen B, Kessler, M et al: Risk factors for bacterial infections in chronic hemodialysis adult patients: a multicentre, prospective survey. Nephrol Dial Transplant 1995;10:377-381.

12. Bonono RA, et al: Risk factors associated with permanent access-site infections in chronic hemodialysis patients. Infect Control Hosp Epidemiol 1997;18:757-761.

13. Ayus JC et al: Silent infection in clotted hemodialysis access grafts. J Am Soc Nephrol 1998;9:1314-1317.

14. Kessler et al: Bacteriemia in patients in chronic hemodialysis. A multicentre prospective survey. Nephron 1993;64:95-100.

15. Bruce J, Sidewy A: Arteriovenous Hemodialysis Access in The Basic Science of Vascular Disease, Anton Sidewy, Bauer Sumpio and Ralph DePalma, 1996.

16. Schwab DP et al: Isolated arteriovenous dialysis access graft segment infection: the results of segmental bypass and partial graft excision. Ann Vasc Surg 2000;14(1):63-66.

17. Miller JH: Partial replacement of an infected arterial graft by a new prosthetic polytetrafluoroethylene segment: a new therapeutic option. J Vasc Surg 1993;17(3):546-558.

18. Gifford RR et al: Management of tunnel infections of dialysis polytetrafluoroethylene grafts. J Vasc Surg 1985;2(6):854-858.

19. McMullen K et al: Salvage of hemodialysis access in infected arteriovenous fistula. Arch of Surg 1991;126(10):130-135.

20. Zibari GB et al: Preoperative vancomycin prophylactic decreases incidence of postoperative hemodialysis vascular access infections. Am J Kidney Dis 1997;30(3):343-348.

21. Stenvenson et al: Standardized surveillance of hemodialysis vascular access infections: 18 month experience at an outpatient, multifacility hemodialysis center- Infect Control Hops Epidemiol 2000;21(3):200-203.

\section{9 \\ Dilatation and Stent Placement in Hemodialysis Fistulas and Prosthetic Grafts}

\section{Turmel-Rodrigues, Tours, France}

Thrombosis occurs roughly 10 times more frequently in prosthetic grafts than in native fistulas and an underlying stenosis is unmasked in more than $85 \%$ of cases for grafts and in almost all cases for native 
fistulas. The literature shows that prophylactic treatment of hemodynamically significant stenosis reduces the rate of thrombosis and prolongs the average use-life of the access. Access infection is the only absolute contraindication to interventional radiology. High pressure balloons (inflatable up to $25 \mathrm{~atm}$ ) can be necessary to abolish the waist of the stenosis. Morbidity rates ranging from 2 to $15 \%$ were reported.

Although the value of self-expandable stents in selected indications cannot be denied, all reports show that they are not the panacea and that their patency is limited because stenosis recurs, either instent or at its extremities. Stents must therefore never overlap major side veins and obviate future access creation. There is a consensus concerning the value of stent placement for rupture control when prolonged balloon inflation fails and for treatment of stenosis recoil.

The success rates are over $90 \%$ for dilation, with frequent resort to stents in central veins. Long-term results after dilation in the largest series are better in forearm native fistulas compared with grafts (best 1 -year primary patency: $51 \%$ vs. $40 \%$ ), with intermediate results in upper arm fistulas. Primary patency rates after stent placement in peripheral locations are not better than after simple dilation, with 1 -year rates ranging from 0 to $23 \%$ for grafts and $20 \%$ in a series of native fistulas mainly located in the upper arm. The published 6-month and 1-year stent primary patency rates in central veins range from 42 to $90 \%$ and from 25 to $70 \%$, respectively.

Radiology achieves results comparable with surgery, with minimal invasiveness and better venous preservation. However wide variations in the results suggest that the degree of commitment of physicians might be as important as the type of technique used.

\section{0}

\section{Surgical Treatment of Thrombosis in Native Arteriovenous Fistulae}

\author{
J.H.M. Tordoir \\ Department of Surgery, University Hospital Maastricht, \\ The Netherlands
}

The incidence of thrombosis of native arteriovenous fistulae (AVF) is 0.2 per patient year. Clotting of the autologous fistula after the first postoperative month is usually caused by anastomotic or outflow vein stenosis, jeopardising blood flow through the fistula. Repetitive puncture of the fistula in the same area with extravasation of blood and hematoma may also lead to fibrosis and stenoses. While an underlying stenosis gradually develops, a significant number of thrombotic failures may be preventable by careful monitoring. Regular clinical examination, measurement of venous pressures, in-line flow methods and determination of dialysis efficacy by calculating $\mathrm{Kt} / \mathrm{V}$, may identify access dysfunction. Duplex scanning and/or fistulography can detect stenotic lesions, which may be amenable to treatment with angioplasty or surgical revision.

\section{Surgical Treatment}

The type of access and the site of thrombosis are important determinants of treatment outcome. Thrombosis may affect the postanastomotic vein segment as result of anastomotic stenosis or may begin at the needle site. When the clot is localised to the anastomosis in radiocephalic and brachiocephalic fistulae, the outflow vein may be patent due to the natural side branches that continues to deliver venous flow.

The surgical treatment options for thrombosed radio- and brachiocephalic fistulae are: 1 . thrombectomy; 2 . thrombectomy with patch plasty; 3. new proximal anastomosis or 4. graft interposition. Surgical exploration of the AV anastomosis is performed under local or regional anesthesia. The proximal and distal artery and the cephalic vein are dissected and vessel loops are admitted. After clamping of the artery, the cephalic vein is longitudinally opened and clots removed from the in- and outflow vessels by Fogarty catheter. When a stenosis is detected (which is usually the case), patch plasty with vein or prosthetic material is employed. In case of stenosis over a long segment, a graft interposition between artery and outflow vein may be necessary. For the common postanastomotic stenosis in radiocephalic fistulae, revision of the anastomosis by mobilizing the cephalic vein and performing a new more proximal anastomosis with the radial artery, is a good option. This is a simple operation with the mean advantage that the already dilated proximal vein can be directly used for cannulation. Thrombosis in brachiobasilic fistulae with transposed veins usually leads to clot propagation into the entire basilic vein. This is the result of the fact that all side branches have been ligation during fistula creation with the result that collateral venous inflow is impossible. An attempt to salvage of a brachiobasilic fistula must be performed within several hours after clotting. Obstructing stenoses are usually the cause for thrombosis and located at the arteriovenous anastomosis and/or at the junction of the basilic with the deep vein. After declotting with a Fogarty catheter, additional repair of these stenoses has to be performed by path plasty or PTA in the operating room.

It may be obvious, that completion angiography after revisional surgery of thrombosed autologous fistulae, is mandatory to detect residual clots and/or stenoses.

\section{1 \\ No abstract received}

\section{2 \\ Mechanical Thrombectomy of Hemodialysis Fistulas and Grafts}

D. Vorwerk

Department of Diagnostic and Interventional Radiology, Klinikum Ingolstadt, Ingolstadt, Germany

Shunt thrombosis is a frequent complication in hemodialysis shunts - particularly in grafts but may be also found in autologous fistulas.

\section{Mechanical Thrombectomy}

Local thrombolysis therapy with several modifications have been described as a suitable approach to shunt thrombosis. More recently, various types of percutaneous mechanical thrombectomy has been proposed but has not yet been fully established in clinical practice. 


\section{Balloon Angioplasty}

Simple balloon angioplasty is the easiest tool to treat small segment thrombosis or small thrombus mass complicating a venous stenosis. Its use is limited to native fistulas since grafts do not show circumscribed thrombosis unless they are treated in a very early stage of thrombus formation.

\section{Combined Surgery and Balloon Angioplasty}

Implant grafts are most frequently declotted using Fogarty balloons after surgical cut-down, combination with balloon dilatation and thrombus cracking for older thrombus formation and combined surgical-radiological intervention.

\section{Aspiration Thrombectomy}

Clot removal may be also performed by us of simple end-hole aspiration catheter of 7 to $8 \mathrm{~F}$. The technique used is similar to arterial thrombosuction; in case a residual flow is present, arterial inflow should be interrupted by digital compression to facilitate aspiration of a clot. It is a simple and cost-effective technique. Some authors use aspiration in combination with thrombolysis by urokinase.

\section{Hydrodynamic Thrombectomy}

Hydrodynamic thrombectomy has been recently introduced to the treatment of acute thrombosis of arteries, bypass grafts and hemodialysis fistulas and grafts. There are three different devices available for that purpose: the $7 \mathrm{~F}$ Hydrolyser (Cordis, Roden Netherlands), the $8 \mathrm{~F}$ SET catheter (HP medica, Augsburg, Germany now OASIS by BSIC) and the $5 \mathrm{~F}$ Angiojet catheter (Possis Inc., USA).

All of them are working with the same principle: they are double lumen catheters with retrograde saline injection from a very small supply lumen that is injected in a larger efferent lumen. Due to the resulting pressure gradient - known as the Venturi effect - between the jet flow and the larger exhaust lumen, the injected fluid instantaneously leaves the vessel via the exhaust lumen thus creating a turbulent flow zone close to the catheter tip and suction. The surrounding thrombus is fragmented by the flow vortex, sucked into the exhaust lumen and removed as a mixture of saline and thrombus through it.

Clinical success was achieved in 39 of 46 technically successful cases $(85 \%)$ in whom the access was used for haemodialysis again or remained patent for at least 1 week after thrombectomy without haemodialysis (three patients).

Cumulative patency was calculated $63 \%$ after 1 week, $57 \%$ after 1 month, $48 \%$ after 3 months, $37 \%$ after 6 and $32 \%$ after 12 months. Although native fistulas showed a trend of better patency after thrombectomy compared to grafts.

\section{Endoluminal Clot Dissolution}

Other than hydrodynamic thrombectomy that removes the thrombus from the body, endoluminal thrombus dissolution tries to break up thrombus formation into ultrasmall pieces allowing capillary passage of the fragments. For clinical purposes the Amplatz clot buster device (Microvena, Minneapolis, USA), the Trerotola nitinol basket (Arrow Inc.) and the Castaneda brush (MTI Inc.) are clinically available. Technical application is very similar to hydrodynamic thrombectomy and depends on the type of shunt that is going to be treated. Applicability is easy and quick since pressured air is available in most angiosuites.

Uflacker compared the Amplatz clot buster to surgical thrombectomy by random in 35 procedures. Success was $89 \%$ and primary patency after 1 months was $47 \%$ for mechanical clot dissolution. He did not find a significant difference in primary patency between surgery and percutaneous technique although primary patency after surgery was better with $75 \%$.

\section{Conclusion}

Thrombosis of haemodialysis fistulae and grafts represents the most frequent complication of hemodialysis access. They are more often found in grafts than in native fistulae. An underlying stenosis or aneurysmatic vein causing flow irregularities are predisposing factors for thrombosis and especially venous stenosis is found in the vast majority of cases. Early reintervention to dilate an underlying stenosis has been shown to reduce the rate of thrombosis. In our daily practice, we try to keep a procedure as simple as possible. In native fistulas, PTA is our first choice that may be combined with hydrodynamic thrombectomy or the clot buster if larger amounts of thrombus are present. In grafts, hydrodynamic thrombectomy is well achievable if the thrombus does not extend into larger brachial veins. If this happens, the clot buster seems to be the better choice.

Applying these strategies, percutaneous techniques have become the approach of first choice in our institution and surgical thrombectomy has been limited to cases were thrombosis is older than a week or failure of percutaneous approach had occurred; thrombolysis has been nearly abandoned from treatment of shunt thrombosis.

\section{3}

\section{Strategy: The Surgeon's Point of View}

V. Mickley

Department for Vascular and Endovascular Surgery, Stadtklinik Baden-Baden, Germany

Introduction: Chronic stenoses of av-fistulae and access grafts can be classified according to their respective locations: (type 1) at the arteriovenous or artery-to-graft anastomosis, (type 2) within the needling segment of vein or graft, (type 3) stenoses at the junction between the superficial vein and the draining deep vein or at the anastomosis between graft and vein. Type 4-stenoses of central (mediastinal or iliocaval veins) will not be discussed here. Different types of anastomosis cause different complications and therefore require a differentiated therapeutic approach.

Fistula or graft thrombosis in most patients is the consequence of undiagnosed or untreated stenosis.

Type 1-Stenoses: In distal radiocephalic fistulae, anastomotic venous stenosis by far is the most frequent long-term complication. If there is some normal vein between artery and stenosis, anastomotic aneurysm will occur. When after months or years of fistula function radial artery and cephalic vein are adequately dilated, ligation of the stenotic segment and proximal re-anastomosis is a simple and safe procedure giving excellent results. Due to the complex morphology of anastomotic stenoses, interventional treatment is demanding. Often excessive pressures have to be applied to dilate the fibrotic segment, and re-stenoses are frequent. Pre-stenotic aneurysm precludes dilatation. Resection of the aneurysm with reconstruction of the artery and proximal re-anastomosis is the treatment of choice.

Arterial anastomotic stenosis due to intimal hyperplasia is an infrequent cause of access graft failure. When only the graft at the anastomosis is stenosed, dilatation can be performed. In most cases, 
however, intimal hyperplasia extends into the artery causing significant narrowing of inflow and outflow. Then surgical resection of the anastomosis with patch angioplasty to the artery and re-insertion of the graft is indicated.

Type 2-Stenoses: Long segmental or multiple string-of-bead stenoses of the punctured vein segment are not so easily treated surgically. Either segmental (vein or PTFE) graft replacement or very proximal re-anastomosis with loss of considerable access length can be performed. Interventional treatment seems a valuable alternative, because it preserves the access site and does not preclude later surgical revision in case of re-stenosis. Stent implantation into the punctured vein segment should, of course, not be considered.

Excessive in-growth of fibrous tissue through puncture holes is the reason for mid-graft stenoses developing months or years after implantation of access grafts. PTA and surgical curettage have been performed successfully. Replacement of the destroyed graft segment, however, seems to be the more logical treatment.

Type 3-Stenoses: In elbow fistulae, stenoses of the cephalic vein close to its junction with the axillary sometimes cause excessive tortuosity and dilation. Interventional treatment must be performed very carefully to prevent cephalic or axillary vein rupture. Stent implantation into the venous confluence will compromise the deep vein and therefore is contraindicated. When dilatation fails or in case of early recurrence, surgical transposition of the central cephalic vein to the proximal brachial vein should be considered.

Venous anastomotic stenosis is by far the most frequent reason for graft insufficiency and occlusion. PTA is the most vein-preserving procedure. High-pressure balloons must be used to achieve complete re-opening of the stenosis, because any residual stenosis will cause early failure. Recoil is frequent and must be treated with stent implantation. In case of early or repeat re-stenosis, graft extension can be considered.

Thrombosis: Both, interventional thrombaspiration and surgical thrombectomy can be used for clot removal from thrombosed access veins and grafts. Immediate and effective (surgical or interventional) treatment of the underlying stenosis according to the above mentioned principles is mandatory for access preservation.

\section{4}

\section{Treatment of Stenosis and Thrombosis in Hemodialysis Fistulas and Prosthetic Grafts: Radiology or Surgery?}

\section{Turmel-Rodrigues}

Tours, France

Access infection is the only absolute contraindication to interventional radiology.

\section{Stenosis}

Central veins Most nephrologists and surgeons agree that dilation must be performed first. The only but major concerns are about the potential overlapping of adjacent vessels by stents.

Native fistulae Surgery should be preferred over dilation for isolated stenoses located within $10 \mathrm{~cm}$ of the wrist in Brescia-Cimino fistulas. Creation of a new anastomosis immediately above the stenosis is a minimally invasive outpatient procedure and does not consume healthy veins. No comparative study between surgery and dilation has ever been performed. However, 1-year primary patency rates after construction of forearm fistulae range from 65 to $84 \%$ whereas the best published rate after dilation is $51 \%$.

In all other cases, dilation should be attempted first until a surgical report proves the contrary (no surgical series available to date in the literature). Surgery must, however, be considered again in cases of repeated early recurrence of stenoses after dilation or stent placement (for example twice in 6 months).

Grafts Surgery has the major drawback of extending the graft farther up the limb and of potentially rapidly reducing the venous capital of the patient. The discussion is not so much whether dilation must be performed first but rather to decide when to go to surgery. In cases of early recurrence of the stenosis after dilation, some teams will place stents whereas others will prefer revision of the venous anastomosis. Redilation every 3 months after stent placement is never justified when simple surgical alternatives are possible.

\section{Thrombosis}

Native fistulae Recent articles have reported excellent results for interventional radiology in experienced hands and are not counterbalanced by the paucity of the surgical literature. Endovascular treatment appears therefore to be preferable, except for flat non-running forearm fistulae. In such cases, a stenosis is typically palpated at the wrist and there are no or few clots above: as for patent fistulae, they should be more durably treated by creation of a new anastomosis.

Grafts DOQI guideline 21 mentions that an $85 \%$ initial success rate must be achieved for both surgery and radiology and most of the recent studies reached this goal.

Two recent randomized studies aimed to demonstrate the superiority of the surgical over the radiological approach in the declotting of grafts. Both were criticized but the DOQI guidelines had already made their choice: given the drawbacks of conventional surgery, higher primary patency rates were assigned to surgery. The $40 \%$ rate suggested at 1 year was achieved but none of the 3 most recent studies since they reported figures ranging from 23 to $26 \%$

In contrast, the suggested $40 \%$ primary patency rate at 3 months for the radiological approach was reached in most recent reports. However, should radiologists be proud of a $60 \%$ failure rate at 3 months? With such figures, how can we not fight for better pre-operative mapping and better training of surgeons, leading to creation of more native fistulae, and how can we not demand enforcement of a stenosis detection programme if a graft is eventually placed? How can we not reproach a surgeon who has placed a graft when the radiologist shows evidence that construction of a more durable fistula was possible?

\section{Basic rules?}

Except for forearm fistula stenoses at the wrist, endovascular techniques have fewer drawbacks and are not clearly less effective than open surgery: they should be used first but they must never compromise future surgery.

Repeated failures of radiology must lead to surgery and vice-versa and the nephrologist's major role is to keep control over any lack of self-evaluation by his colleagues.

The level of dedication of local physicians is essential to determine the best policy: not all the techniques are equal or at least they are not standardized and the results are operator-dependent.

However it can be said in 2001 that there is an unacceptable loss of opportunity for patients who are dialyzed in centers not working in close cooperation with interventional radiologists. 


\section{Other Complications and Types of Vascular Access}

\section{5}

\section{Distal Ischemia: Radiological Approach and Treatment}

\author{
A. Raynaud \\ Hôpital Européen Georges Pompidou, \\ Clinique Alleray-Labrouste, Paris, France
}

Distal ischemia is a severe and frequent complication of angioaccess for hemodialysis. Three mechanisms which are often associated may cause such a complication: increase in venous pressure, steal syndrome and arterial lesions. We will focus on the two latter mechanisms. The diagnosis of distal ischemia is mainly based on clinical features. Ischemia may present under various forms:

- acute ischemia with neuromuscular deficiency;

- chronic ischemia with hand pain, numbness, paresthesia, progressive weakness and sometime trophic changes and gangrene;

- ischemic monomelic neuropathy with severe sensitory and motor nerve dysfunction and very moderate improvement after revascularisation or access closure.

Non-invasive examination such as plethismography, ultrasonography and pulse oximetry may help the diagnosis. However, angiography remains necessary in most patients. It provides mapping of arterial lesions and images flow.

\section{Steal Syndrome}

The blood pressure into the access is far lower than into the arteries. Thus the blood flow is diverted by the access.

When the access is anastomosed on the brachial artery, the flow in the distal part of the brachial artery, downstream from the anastomosis, is dramatically modified. It can be antegrade in systole and diastole, antegrade in systole and retrograde in diastole or retrograde in both systole and diastole. Nevertheless, in the last two cases supply to the hand is nevertheless often sufficient and comes from collaterals originating mainly from the proximal part of the brachial artery and the deep brachial artery.

In some cases, supply to the hand is not sufficient and distal ischemia occurs. Vascular radiology has no role in the treatment of such cases but it is important for preoperative screening. It guides the choice of surgical technique: it rules out associated arterial lesions.

Accesses created at the wrist are fed by the proximal and the distal radial arteries. In the latter, the flow is retrograde because of it is supplied via the ulnar and the palmar arches. In such cases steal syndrome occurs only when the ulnar artery is stenosed or occluded. The flow in the distal radial artery may then be retrograde and cause steal syndrome. In such cases treatment of the hand ischemia is by reopening of the ulnar artery by PTA. In rare cases when PTA is not feasible ligation or endovascular closure of the distal radial artery may cure the ischemia.

\section{Arterial Lesions}

Stenoses and occlusions of arteries are the most frequent causes of distal ischemia in patients under hemodialysis. Lesions may be proximal or distal to the arterial anastomosis.

\section{a. Lesions Proximal to the Arterial Anastomosis}

In cases of brachial access, the lesions upstream from the arterial anastomosis can be located from the subclavian artery to the arterial anastomosis. Treatment by PTA is very simple. It causes an increase in flow in both access and distal arteries, and is often effective in treating ischemia. Such lesion should absolutely be ruled out before thinking about steal syndrome.

In cases of Brescia Cimino access, PTA of stenoses located from the subclavian to the brachial artery is also very simple and effective. However the lesions may also involve radial and ulnar arteries.

Angioplasty should be considered for stenosis of the proximal part of the radial artery with the aim of treating distal ischemia even when the flow in the distal radial artery is retrograde because it may overcome steal syndrome. The high flow due to the access after dilatation, results in very rapid and favorable healing of the parietal damage, it also decreases the risk of thrombosis. Thus, short and long term morphological results are often satisfactory. However the results are uncertain for hand ischemia. PTA always clearly increases access flow but the increase in flow in the distal radial artery is unreliable and often moderate.

Lesions of the ulnar arteries should also be considered as proximal lesions in the case of Brescia Cimino access. In contrast to the radial artery, the ulnar artery feeds the hand before the access and thus the treatment of such stenoses or segmental occlusions is, in our experience, very effective on hand ischemia.

\section{b. Lesions Distal to the Arterial Anastomosis}

For accesses anastomosed on the brachial artery, distal lesions may be located on the distal brachial artery or on forearm arteries.

In cases of stenosis of the brachial artery distal to the inflow, effectiveness for hand ischemia will depend on the flow in the artery. When the flow is clearly antegrade, PTA will improve supply to the hand. However, PTA is contra-indicated when the flow in the distal brachial artery is retrograde because it will worsen steal syndrome. When the distal brachial artery is completely occluded the effectiveness of recanalization on distal ischemia cannot be predicted.

Lesions of both ulnar and radial arteries are necessary to cause distal ischemia, one of these two arteries being sufficient to supply the palmar arches. Lesions are often so extensive that no PTA or surgical treatment can be considered.

\section{Conclusion}

Thorough knowledge of mechanisms of distal ischemia is necessary for pretreatment screening and to guide the choice of treatment. PTA of arterial lesion is the treatment of choice for many patients. Because of the high flow induced by the access the morphological results of arterial PTA are often very satisfactory. PTA can thus be attempted in patients with severe and extensive disease. Treatment of distal ischemia by arterial occlusion is a paradox but is effective when steal syndrome has been identified. 


\section{6}

\section{Ischaemia and Surgical Treatment}

\section{A. Bakran \\ University of Liverpool, The Royal Liverpool University Hospital, Liverpool, UK}

Hand ischaemia, usually described as 'steal syndrome', is variously thought to afflict between $1-8 \%$ of patients after arterial venous fistula creation [1-3]. Whilst it is acknowledged that it can occur following a radiocephalic AV fistula formation, it is, however, usually more frequent after an AV fistula which uses the brachial artery as the inflow vessel. Symptoms can vary from complaint of coldness of the hands, cyanosis of fingers, to pain and numbness in the hand and finally to outright loss of sensation with paralysis of hand function (monomelic neuropathy), which is a prelude to ischaemic ulceration and gangrene. Diabetics and those with severe peripheral vascular disease are of special risk of development of steal syndrome.

Diagnosis of ischaemia should be based is based not only on symptoms but also on objective findings. The simplest method of assessment of steal syndrome is the measurement of the wrist brachial pressure index (WBPI) which is similar to the ankle brachial pressure index in patients with peripheral vascular disease of the lower limbs. The WBPI should be at least ' 1 ' in the normal patient prior to fistula creation but if it falls to below 0.6 , then symptoms of steal syndrome seems to occur [4]. This test can be performed on the ward using a simple hand held Doppler device and a sphygmomanometer. Other tests include digital plethysmography and pulse oximetry $[5,6]$. Ironically, pressures in diabetics may be inappropriately high due to incompressibility of the diseased arteries in the forearm. All the above are non-invasive tests but arteriography will reveal the extent of steal in such patients, since, in severe cases, very little contrast will be seen to fill the radial and ulnar arteries leading to the hand.

The underlying cause of ischaemia is clearly diversion of blood flow up the arteriovenous fistula and away from the hand. Therefore, management of steal syndrome involves mechanisms to reduce the fistula blood flow so that more blood flows distally to the hand. In order to achieve adequate dialysis, a blood flow through a fistula should be a minimal of $500 \mathrm{ml}$ per minute, although brachial fistulae of whatever variety often have flows exceeding 1 litre per minute. Thus, any technique used to reduce fistula flow should reduce blood flow down to a range between $500 \mathrm{ml}$ to $800 \mathrm{ml}$ per minute and intraoperative method of assessing blood flow should be utilised to confirm adequacy of flow reduction. Techniques used to reduce blood flow are as follows:

1. Avoid a long anastomosis length at time of AV fistula creation since this will increase the risk of excessive blood flow diversion.

2. Banding of autologous or prosthetic graft AV fistulae by:

(a) Plication of fistula using sutures

(b) Using a tapered graft segment to interpose between artery and fistula vein

(c) Suture a short narrowing segment of PTFE around fistula

(d) DRIL procedure - insert a bypass (vein or prosthetic graft) from brachial artery above the fistula to brachial artery below the fistula and then ligating the brachial artery just distal to the AV fistula anastomosis.

(e) Closure of the AV fistula - in order to salvage the hand in severe cases of ischaemia or instances of ischaemic monomelic neuropathy.
The problem with banding is assessing how narrow the segment diameter should be and for what length the fistula should be narrowed. The danger is thrombosis or inadequacy of fistula flow if the surgeon is overzealous. It is, therefore, fundamental that an intra operative technique is used to assess the adequacy of the flow up the fistula and adequacy of flow reduction to overcome ischaemia. The DRIL procedure involves ligating a normal brachial artery, which may not be the best option for long term.

In conclusion, ischaemia resulting from an arteriovenous fistula can be a devastating complication for the patient. Assessments of finger blood flow intra-operatively or certainly post-operatively following AV fistula formation will highlight the risks of steal syndrome developing and measures should then be taken early to prevent the long term consequences of this complication.

\section{References}

1. Odland MD, Kelly PH, Ney AL, Anderson RC, Bubrick MP: Management of Dialysis Associated Steel Syndrome Complicating Upper Extrematory Arteriovenous Fistulas: Use of Intra Operative Digital Photo Plethysmography. Surgery 1991;110:664-667.

2. Rivers SP, Scher LA, Veitch FJ: Correction of Steal Syndrome Secondary to Haemodialysis Access Fistulas: A Simplified Qualitative Technique. Surgery 1992;112:593-597.

3. Bussell JA, Abbott JA, Lim RC: A Radial Steal Syndrome with Arteriovenous Fistula for Haemodialysis. Ann Int Med 1971;75:387-394.

4. Bakran A: Diagnosis and Surgical Treatment of Ischaemia. Dial J 1999; 18(66):259-260.

5. Halevy A, Halpern Z, Negri M, Hod G, Weissgarten J, Averbukh Z, Modai D: Pulse Oximetry in the Evaluation of the Painful Hand after Arteriovenous Fistula Creation. J Vasc Surg 1991;14:437-439.

6. Dally P, Brantigan CO: Plethysmography and the Diagnosis of the Steal Syndrome Following Placement of Arteriovenous Fistulas and Shunts for Haemodialysis Access. J Cardiovasc Surg 1987;28:200-203.

7. Sivanesan S, Bakran A, Howe TV: Characterising Flow Distribution in AV Fistulae for Haemodialysis Access. Nephrol Dial Transplant 1998;13: $3108-3110$.

8. West JC, Bertsch DJ, Peterson SL, Gannon MP, Norkus G, Latsha RP, Kelley SE: Arterial Insufficiency in Haemodialysis Access Procedures: Correction By 'Banding' Technique. Transplant Proc 1991;23:1838-1840.

9. Schanzer H, Skladany M, Haimov M. Treatment of Angioaccess Induced Ischaemia by Revascularisation. J Vas Surg 1992;16:861-866.

27

\section{No abstract received}

\section{8}

\section{Complicated Vascular Accesses}

\section{J.R. Polo \\ Vascular Access Unit, Hospital General Universitario Gregorio Marańon, Madrid, Spain}

Unusual vascular access have to be performed in patients without possibility of peritoneal dialysis in which a combination of the following circumstances are present.

1. Obstruction of thoracic central veins occasioned by pacemakers, cardiothoracic surgery, previous dialysis catheters etc. 
2. Obstruction of upper and/or lower limb arteries

3. Severe cardiac failure

4. Wasting of arteries and veins due to multiple previously failed angioaccesses

Most of these access have to be performed using grafts or permanent catheters. In these patients it is not possible to perform: (a) upper limb accesses autologous or prosthetic; (b) jugular or subclavian vein cuffed permanent catheters.

\section{Non-Dilatable Subclavian Vein Occlusion}

A brachiojugular graft can be placed at the external or internal jugular vein in cases of subclavian vein occlusion (Haimov $M$. Surgery 1982;92:109-110, Polo JR et al: Annals of Vascular Surgery 2001;15:553-556). Puncture sites can be performed at the upper arm part of the graft. We have an experience with 54 cases. Periprosthetic infection was very low (3.7\%) and secondary patency reached almost $60 \%$ at 5 years. The commonest complication was graft-vein stenosis as usual with all kind of grafts. Other alternatives described for intrathoracic vein occlusion had been right atrial bypass (El-Sabrout RA, Duncan JM et al: J Vasc Surg 1999;29:472-478) and axillary artery-to-axillary vein grafts (Garcia-Rinaldi et al: Am J Surg 1978;135:265-268. McCann RL: J Vasc Surg 1996;24:457-462). Axillo-femoral grafts have also been employed (Rueckerman et al. ANNA Journal 1991;18:567-571)

\section{Vena Cava or Right Atrial Occlusion}

Right atrial thrombosis is being seen more and more frequently due to the over-use of permanent catheters (Am J Kidney Dis 2001;38:631-639). In absence of arterial pathology a femoro-femoral graft can be placed. A experience with 37 cases and 4 years $50 \%$ cumulative patency have been reported (Korzets A et al: Nephrol Dial Transplant 1998;13:1215-1220). In a personal experience with 17 cases, periprosthetic infection was $18 \%$ (infection rate was $8 \%$ in our experience with upper limb grafts) and a high rate of venous stenosis was found. This last complication could be due to the fact that most of these patients had previous femoral catheters for many time placed to overcome complications in previous upper arm accesses. Subcutaneous transposition of the superficial femoral vein after anastomosis with the distal femoral artery is another possibility (Petaen H. Proceedings of the VAS London meeting 2001) as was described by P Bourquelot. Lower limb edema has been frequently described with this technique. In cases in which arterial pathology discourage a lower limb vascular access a transfemoral permanent catheter of at least $50 \mathrm{~cm}$ in length can be placed. A reasonable experience with 41 of these catheters have been reported (Zaleski GX: AJR 1999;172:493-496). We have experience with five of these catheters used for more than 2 years. Thrombotic occlusion of the catheter is quite frequent. Thus, we advise to place all these patients on oral anticoagulation. The deep circumflex iliac vein as been used by cut-down as another alternative entry port (White SA et al Nephrol Dial Transplant 2000;15:244-245)

\section{Both Superior and Inferior Vena Cava Occlusion}

An arterio-arterial graft can be placed (Scholz $\mathrm{H}$ et al: In Henry ML Proceedings of Vascular Access for Hemodialysis VI, Miami, Precept Press, 1999, pp 255-262). The patients have to be place on anticoagulation because the flow can be very low in the upper arm (below $200 \mathrm{ml} / \mathrm{m}$ ) and the long-term patency of dialysis grafts have been found to be flow dependent with the critical flow below $800 \mathrm{ml} / \mathrm{m}$.
Otherwise, a low flow-long time dialysis should be necessary to avoid recirculation. Others alternatives are translumbar permanent catheters (Lund GB et al: Am J Kidney Dis 1995;25:732-737. Biswal R et al: Cardiovas Interven Radiol 2000;23:75-78; Azizkhan RG et al: J Pediatr Surg 1992;27:165-169), or transhepatic permanent catheters (Po CL et al: Am J Kidney Dis 1994;24:590-591. Bergey EA. Pediatric Radiology 1999;29:42-45). We have experience with a case of translumbar Permcath catheter working for 6 months with good dialysis flow. As the length of femoral catheter have to be very long a translumbar approach should probably preferable in cases of inferior vena cava catheters.

Some patients with superior vena cava occlusion have been treated by recanalization of the occluded venous segment through a transfemoral route and placement of a yugular catheter guided to the right atrium with a loop snare. (Funaki B et al: Radiology 2001;218:471-476). Transmediastinal puncture, using a suprasternal approach, of the innominate vein for atrial catheter placement have been also published using a US guidance (Lau TN et al: J Vasc Interv Radiol 2001;641-645). The superior caval vein can be approached through a second intercostal MR imaging guidance catheter placement (Sewell PE et al. AJR 2001;176:233-234). Direct placement of a catheter in the superior vena cava under local anesthesia has been also published (Archundia AG: Nephrol Dial Transplant 2002;17:134-136)

\section{9 \\ Haemodialysis Access and Cardiovascular Outcomes in End-Stage Renal Disease}

\author{
R.N. Foley \\ Hope Hospital, Salford, UK
}

Cardiovascular disease is virtually a sine qua non in dialysis patients, with attributed mortality rates orders of magnitude higher than in the general population. Recent estimates from the United States suggest that a typical patient starting dialysis therapy has a probability of developing a major cardiovascular event of at least $50 \%$ in 5 years, with extremely high subsequent mortality rates. Congestive cardiac failure is easily the commonest reason for cardiovascular admission, followed by peripheral vascular disease, acute coronary syndromes and stroke. Uraemia, and its therapy, is a state of extreme haemodynamic, thrombotic, inflammatory and endothelial stress to an inherently dysfunctional cardiovascular system. Dialysis access can contribute to all these mechanisms in different ways. Unfortunately, there has been relatively little systematic study, to date, regarding the impact of dialysis access and cardiovascular. It is known for example, that the presence of a native fistula leads to left ventricular dilatation, which itself clearly predisposes to cardiac failure and death. Synthetic grafts and central lines, on the other hand, are more likely to offer the threats of inflammatory, thrombotic and endothelial stress. Recent observational work, again from the United States, suggests that mortality rates are higher, after case-mix adjustment, for patients with artificial grafts or central venous catheters. Preliminary analysis suggests that central venous catheters are associated with a higher incidence of acute coronary syndromes, but an equivalent incidence of cardiac failure as native fistulas, suggesting that the thrombotic/inflammatory risks of foreign materials may outweigh the haemodynamc risk of native fistulas. 
30

\section{Anterior Chest Grafts}

M.A. Yerdel

Ankara University Medical School, Department of General Surgery, Ankara, Turkey

Our experience with anterior chest grafts (ACG) by using infraclavicular axillary or internal jugulary vein (IJV) as outflow vessels in patients with wasted arm veins is presented.

\section{Patients and Methods}

Patients with wasted upper extremity fistula sites who were candidates for a femoral graft or a permanent catheter and/or otherwise 'non-hemodialysable' with conventional methods entered the protocol and prospectively analyzed. Patients were followed-up by monthly intervals

\section{Results}

Between July 1999 to January 2002, 22 patients were implanted with 23 ACG (Mean age: 61) (M/F: 7/15). All had bilateral subclavian vein catheterization history and most had previous IJV catheterizations. Mean number of previous access procedures was 8.4 / patient. Presence of a suitable vein was secured by angiograms in $21 / 23$. Subclavian \& innominate veins were assessed by venography. IJV's were assessed by late phase carotid angiograms. All but two operations were done under local anesthesia. Through a transverse infraclavicular incision axillary vessels are encircled medial to the insertion of the pectoralis minor without dividing it. IJV is exposed through a vertical incision between the heads of sternocleidomastoideus muscle. Ansa cervicalis and omohyoideus muscle may come into view and should be saved. IJV is controlled with a side clamp. Once inflow and outflow vessels are controlled, a $6 \mathrm{~mm}$ graft is tunneled through the anterior chest wall as appropriate. Both anostomoses are done in an end to side fashion by running 5/0 polypropylene. 19 Cases were implanted with a heparin bonded polycarbonate/urethane graft (Chronoflex, CardioTech Int., U.K.) and 4 with stretch/standard walled ePTFE (W.L. Gore-Tex, U.S.A.). The outflow vein was IJV in 15 (9 crossover and 6 ipsilateral loop) and axillary vein in 8 (3 crossover and 5 ipsilateral loop). Inflow vessel was infraclavicular axillary artery in all but 1 patient. There were two early complications. Face edema in a patient with distally ligated IJV with contralaterally occluded IJV was spontaneously subsided. Hemiparesis in another patient also completely resolved in a few days. One late complication, pseudoaneurysm at puncture site (ePTFE) required graft interposition. No steal, infectious complication or permanent neurologic deficit occurred. The mean time interval between graft implant to first dialysis was 12 days in polyurethane (shortest $16 \mathrm{~h}$ ) and 25 days in ePTFE grafts. All but one patient were dialyzed $3 /$ week. Thrombotic episodes were managed by thrombectomy in all but 1 in whom thrombolysis was done. Patients were followed up between 1-32 months. Three, 6 and 12 months 'actual' assisted patencies were 90, 60 and $40 \%$ respectively. Longest patent graft is still being dialyzed at $32 \mathrm{nd}$ month. Six patients died of irrelevant causes during the follow-up. Nine patients lost their grafts to irreversible thrombosis (subsequently 6 had a perm-cath and 3 had femoral grafts). One graft loss occurred in an extremely hypotensive patient. All (n:5) early graft losses ( $<6$ months) occurred in patients with venographically proven compromised venous outflow (partially patent veins).

\section{Conclusions}

Use of infraclavicular axillary or IJV as outflow vessels in graft fistulas did not raise much interest owing to the complicated nature of the procedures. Currently only single series is available from DUKE University U.S.A. [1]. Patency rates in graft fistulas is affected by many factors and compromised venous outflow is of uppermost importance. Almost all of our cases having good outflow veins remained patent more than 6 months and a $60 \% 6$ and $40 \% 12$ months patency rates are understandable as most graft losses occurred in patients having partially occluded outflow veins. The question whether such occlusions must be stented preoperatively remains valid. We showed the feasibility of local anesthesia. This is a step forward eliminating the need for general anesthesia in these vulnerable patients. Steal has never been a problem as proximal axillary artery is very rich in collateral flow. In fact these operations were used as a solution for the steal problem in more distally located fistulas [1]. Skin overlying the chest wall is easy to cannulate, it is not very sensitive and patient comfort is even better than the arm fistulas. Our infection rate was $0 \%$ and the importance of this fact can not be overemphasized as the other alternative in similar cases is thigh fistulas which are associated with unacceptable infection rates. In conclusion this series provides data pointing out the success, feasibility and low complication rate of ACG. This is especially true when there is not a venous outflow problem. Therefore ACG using central veins are here to stay and larger series with proper follow-up will establish their definite role in the management of complicated access patients.

\section{Reference}

1. McCann RL. Axillary grafts for difficult hemodialysis access. J Vasc Surg 1996;24:457-462.

\section{Central Vein Catheter Access and Vascular Access Strategy}

\section{1 \\ Long-Term Catheter for Hemodialysis: Predicting and Outcome \\ J. Pengloan \\ Unité d'Hémodialyse Chronique, CHU Bretonneau, Tours, France}

Long-term catheters (LTC) are essential for the management of chronic hemodialysis patients (CHP); an increasing number of CHP are treated with LCT. A high morbidity rate is related to LCT; catheter malfunction and infectious complications limit the survival rate. Long-term patency rate of LTC is quite different in USA and Europe; in USA reported catheter survival rate were $33 \%$ at 90 days or $55 \%$ 
at 180 days; the patency estimated by the DOQI panel was 12 months. In Europe the catheter survival rate could reach $50 \%$ and $65 \%$ at 3 years.

Catheter malfunctions observed after placement are related to misplacement (ideally tips have to be placed in the atrium) or stricture; catheter are to be replaced. Thrombosis and fibrin sheath can occur during the usage of the catheter; corrective measures are based on fibrinolytic treatment and radiological intervention in case of failure.

Reported catheter-related bacteremia rate ranged from 1.4 to 5.5 per 1,000 catheter-days with up to $40 \%$ of severe complications and a high mortality rate.

Catheter-related infection is a 4 -stage process well described by Costerton: bacteria are firstly introduced into the lumen during the procedures of connections, secondly they adhere to the inert material and lost their properties; they grow in a protective biofilm. From microcolony to an another microlony catheter can be entirely colonised. Fibrin helps the growing of bacteria. But whatever the stage bacteria can be launched into the bloodstream where they return virulent and lead to septicaemia and distal abscesses

In 2/3 of cases Staphylococcus sp are causative pathogen and in $2 / 3$ of cases the infection lead to the removal of the catheter.

The prevention of catheter-related infection is based on rigorous respect of 'Universal Precautions' to prevent the introduction of bacteria during the procedure of insertion and during handling of catheter. Different types of filling solution have been proposed to suppress adhesion of bacteria; antibiotic-lock solution such as citrategentamycin have to be avoided because toxicity and risk of bacterial resistance. Utilisation of Citrate or Taurolidin, which is an antiseptic, are promising; however long-term side effects such as toxicity or selection of resistant pathogens could not be excluded.

Early detection of intraluminal colonisation and therefore early treatment could prevent catheter-related bacteremia. In our institution regular surveillance of intraluminal colonisation has been instituted. The contents (i.e. heparin and the 1 st cc of blood) of each branch of the dialysis-catheter are cultured monthly before starting a dialysis session. If the culture is positive, cultures of the catheter contents are repeated before the next two sessions. If the three consecutive cultures are positive, intracolonisation is confirmed and treatment is initiated. Treatment is commenced immediately if there are systemic signs. It is based on:

- treatment of the patient with systemic treatment: Cefazolin ${ }^{\circledR}$ and Netromycin ${ }^{\circledR}$ by IV route at the end of the session, with half a dose of each antibiotic through each lumen

- treatment of the catheter with thrombolytic treatment: Urokinase before 2 or 3 consecutive sessions; each lumen is filled with an antibiotic solution of $100 \mu \mathrm{g} / \mathrm{ml}$ in sodium heparin (netromycin or vancomycin according to antibiogram).

Vancomycin is reserved as a second line treatment and for methicillin-resistant Staphylococcus.

In a prospective study totalling 36 catheters and 19,208 catheterdays the catheter-colonisation rate was 0.68 per 1,000 catheter-days and the catheter-related bacteremia was 0.21 per 1,000 catheter-days. A patency rate of $65 \%$ at 36 months was achieved. Only one catheter was removed for infectious cause (persistent catheter colonisation with fever despite intensive and appropriate treatment).

Long-term survival of LTC can be achieved; strict application of Universal precautions of asepsis associated with early detection and treatment of intraluminal colonisation could explain our good results. But to improve catheter survival rate we furthermore need technological improvement of hubs but also progresses in pharmacological and in bacteriological knowledge.

\section{2 \\ Central Venous Catheters: Different Types of Locking Solutions}

\author{
J.B. Ponikvar \\ Department of Nephrology, University Medical Center, \\ Ljubljana, Slovenia
}

In interdialysis period hemodialysis catheters are not in use. After insertion and after each dialysis they are usually rinsed with saline and locked with concentrated unfractioned heparin solution. The volume of locking solution is adjusted to the volume of catheter. This standard approach can cause significant systemic heparinization.

In the early nineties we have observed bleeding in several patients in intensive care units, after performing heparin free dialysis, and after femoral catheters were locked with heparin. This observation has lead us to perform a pilot study to measure acivated partial thromboplastin time before and 15 minutes after the insertion and the locking the catheter with heparin. Blood samples for coagulation test have been taken from peripheral vein. Significant systemic heparinization was found. After that randomized study was performed, comparing three different locking solutions for single lumen temporary hemodialysis catheters: heparin, $4 \%$ trisodium citrate and polygeline $\left(\right.$ Hemaccel $\left.{ }^{\circledR}\right)$. We have found no difference in the rate of catheters malfunction or volume of clot aspirated from the catheters [1]. Locking the single lumen temporary dialysis catheters with $4 \%$ trisodium citrate became a standard practice at our center after 1996. Several hundreds of catheters were locked with citrate and no adverse effects due to locking solution were observed. During the insertion and citrate locking afterwards the patiens were recorded on ECG monitor and no significant adverse influence on cardiac rhythm was observed.

There were reports on sudden death when catheter were locked with high concentrated citrate solution (46.7\%). Local hypocalcemia at the catheter tip, in the vicinity of right atrium, could be the reason. We believe it's advisable to use low citrate concentration for catheter locking.

The advantages of citrate locking instead of heparin are: the absence of systemic anticoagulation, low price, knowledge of the precise filling volume of the catheter in not critical. Volume of locking solution can be safely increased by 1 or $2 \mathrm{ml}$, without causing systemic anticoagulation or other clinically relevant side effects. This could be of great advantage in busy dialysis centers which use several types of catheters of different lengths.

Another study was performed at our center comparing heparin and $4 \%$ trisodium citrate as the locking solution for permanent silastic catheters. In this setting heparin was found to be slightly superior to citrate as concerns catheter functioning time. So in situation of permanent silastic hemodialysis catheters we lock them with heparin and use citrate only in the patients at risk of bleeding [2].

We have also used $4 \%$ human albumin as a locking solution in a patient treated by plasma exchange for a longer period, without adverse effects.

Karaaslan et al. reported a risk of serious bleeding in Dual-Cath ${ }^{\circledR}$ heparin locking and suggest use of sodium citrate, polygeline or urokinase as the alternative to heparin [3].

There was a study of locking double lumen Quinton Permcath catheters with tissue plasminogen activator, reccomending it as a useful alternative to heparin. High price of tissue plasminogen activator will probably be the limiting factor of this approach [4] 
Another interesting approach has been the attempt to add antibacterial properties to the anticoagulant properties of the locking solution. Neutrolin ${ }^{\mathrm{TM}}$ (Biolink, Norwell, MA) is a mixture of citrate and antibiotic taurolidin [5]. It is originally prescribed for a special type of vascular access, Dialock ${ }^{\circledR}$ system, but providing acceptable price it could be applied for other types of catheters, too. There was a report on the use of low molecular weight heparin for Dialock ${ }^{\circledR}$ system lock in some patients.

In conclusion, standard heparin locking of hemodialysis catheters can expose the patient to the significant risk of bleeding. There are several alternatives to heparin reported like citrate in different concentrations, polygeline, human albumin, tissue plasminogen activator, mixture of citrate and antibiotic taurolidin. The policy at our Department is to lock all single lumen temporary polyurethane catheters with $4 \%$ trisodium citrate. Silastic catheters are locked with heparin, except in the patients at risk of bleeding, where $4 \%$ trisodium citrate is used. New special solution, combining citrate and antibiotic taurolidin, could be further step in reducing both major complications of hemodialysis central venous catheters, thrombosis and infection, without exposing the patient to the risk of systemic heparinization.

\section{References}

1. Buturović J, Ponikvar R, Kandus A et al: Filling hemodialysis catheters in the interdialytic period: heparin versus citrate versus polygeline: a prospective randomized study. Artif Organs 1998;22:945-947.

2. Ponikvar R, Urbančič A, Buturović-Ponikvar J: Heparin versus 4\% trisodium citrate as a locking solution for silastic jugular hemodialysis catheters. Proceedings from Second Slovenian Congress on Nephrology with international participation. Ljubljana 2000;171-174.

3. Karaaslan H, Peyronnet P, Benevent D et al: C. Risk of heparin lockrelated bleeding when using indwelling venous catheters in haemodialysis. Nephrol Dial Transplant 2001;16:2072-2074.

4. Schenk P, Rosenkranz AR, Wölfl G et al: Recombinant tissue plasminogen activator is a useful alternative to heparin in priming Quinton Permcath. Am J Kidney Dis 2000;35:130-136.

5. Sodemann K, Polaschegg H, Feldmer B: Two years experience with Dialock ${ }^{\circledR}$ and CLS (Neutrolin) (A new antimicrobial lock solution). Blood Purif 2001;19:251-254.

\section{3 \\ Stenosis and Thrombosis of Central Veins \\ V. Mickley \\ Department for Vascular and Endovascular Surgery, Stadtklinik Baden-Baden, Germany}

Introduction: Central venous obstruction arising from longterm or repeat catheterizations for haemodialysis access is a frequent problem in ESRD patients. If the draining vein of a functioning vascular access is obstructed, venous hypertension with arm swelling, pain and ulceration will occur. Surgery (patch angioplasty or bypass procedures) can be difficult and hazardous in these often multimorbid patients. Thus it seems necessary to define the relative value of interventional treatment (percutaneous angioplasty and stent implantation) in comparison to surgical reconstruction of central venous obstructions in haemodialysis patients.

Patients and Methods: From 1993 through 2001, 39 haemodialysis patients ( $3 \%$ of all access procedures, $5 \%$ of all access patients) presented with symptomatic mediastinal vein obstructions (disabling arm swelling in 39, distal ulcerations in three) on the side of a functioning access. Percutaneous angioplasty and stent implantation was possible in 25 patients $(64 \%)$. When interventional procedures were unsuccessful, veno-venous bypasses were implanted (6 patients, $15 \%$ ), or access ligation was performed ( 8 patients, $21 \%$ ). All patients were followed-up prospectively.

Results: Following stent implantation one asymptomatic pulmonary stent embolism (4\%) and two stent misplacements (8\%) were documented. The latter were successfully treated with another stent. In the surgical group, one patient died at eight weeks due to late complications of a cephalosporine-associated Lyell syndrome. Patency rates (life table-method) were not significantly different among the groups:

\begin{tabular}{llllll}
\hline \multirow{2}{*}{ Patency } & \multicolumn{2}{l}{ Primary } & & \multicolumn{2}{c}{ Secondary } \\
\cline { 2 - 3 } \cline { 5 - 6 } & 1 year & 2 years & & 1 year & 2 years \\
\hline Stents & $78 \%$ & $62 \%$ & & $96 \%$ & $88 \%$ \\
Bypasses & $83 \%$ & $67 \%$ & & $100 \%$ & $83 \%$ \\
\hline
\end{tabular}

Conclusion: For central venous stenoses in haemodialysis patients, angioplasty and stent implantation can be recommended as a safe and durable means to restore patency. Still there is a role for bypass surgery in severely symptomatic central venous occlusions when stent implantation is impossible or has failed. In high-risk patients, however, access ligation should be performed.

34 The Dutch Approach: Towards A National
Multidisciplinary Vascular Access
Surveillance Program

M. van Loon, W.A.M.A. van der Mark, P.J. Blankestijn,

R.M. Huisman, F. van der Sande, J.H.M. Tordoir, J.J. Zijlstra

The Netherlands

Background: In previous studies we showed that active vascular access surveillance results in a reduction of thrombosis rate, meeting the quality of care standard of a thrombosis rate of $<0.5$ per patientyear recommended by The Taskforce of the NKF/KDOQI. As a consequence the number of interventions because of stenosis without thrombosis increases. The Dutch Kidney Foundation (Nierstichting Nederland) initiated a program of Vascular Access Care. A multidisciplinary national taskforce including nephrologists, radiologist, vascularsurgeons and vascular access nurses was founded.

Aims of the project 'The overall objective of the program Vascular Access Care' is to comply nation wide with quality of care standards of the KDOQI, thereby improving quality of live. Our primary objective is to reduce thrombosis rates and secondary objective was to increase the placement of native AV fistulas. Methods 'Three Vascular Access Coordinators' are instituted. Their tasks are: Define a national consensus and protocols on vascular access care (based on the guidelines of the NKF/KDOQI and in the near future the European Guidelines). 
Protocols include:

- well organized patient education and patient information

- training of all staff involved in patient care

- database to collect all relevant patient data

- weekly meetings to discuss complications and to evaluate interventions.

Primary objective is reached by: Introduction of a well defined surveillance program for early identification of imminent dysfunction.

Timely referral for additional diagnostic and corrective interventions (mainly PTA) when predetermined levels of VP or flow are reached.

Secondary objective: Program aimed at preservation of veins, proper preoperative diagnostic procedures, timely placement of permanent access Identification of failure to mature.

Effect measures:

- number of native AV fistula

- thrombosis rate

- number of intervention

- catheter days (= day that patient had a temporary catheter because of shunt failure).

Conclusion: By this national initiative we aim to implement vascular access care, which will allow us to reach the quality of care standards of the KDOQI taskforce in as many as possible dialysis units. The central role of the Vascular Access Coordinator in this program of maintenance is evident. The standard electronic database will collect the outcome parameters of this program.

\section{5}

Nursing Care Documentation for Monitoring AV Fistulas in Hemodialysis Patients: Better Long Life Expectancy?

\section{Malovrh, A. Semolic, Z. Pogorevc, M. Pecik-Gostincar \\ Department of Nephrology, University Medical Center \\ Ljubljana, Ljubljana, Slovenia}

Vascular access procedures and their subsequent complications represents a major cause of morbidity for chronic hemodialysis patients. Besides the successful vascular access creation and adequate access maturation, careful monitoring of vascular access function during hemodialysis treatment would be very important. First sign for any of frequent complications (thrombosis, stenosis, low AVF flow, recirculation etc.) could indicate need for early intervention and prevention of AVF loose. Nurses are the key in the recognition of such first signs.

A complete documentation enabling systematic and continual nursing care of AVF was prepared for short or long term monitoring of all changes. Documentation consists of four documents:

a. Document No 1: basic information about the patient: age, primary renal disease, sex, date of AVF construction, location, when AVF was first used, a brief description of AVF, and short description of medical documentation about AVF.

b. Document No 2: drawing of AVF-artery and fistula vein, place of anastomosis, line of fistula vein over the whole AVF arm, marked punctures spots; enclosed is also a photo of the AVF and table for registering needless should be used and some special details of puncture of this concrete AVF.

c. Document No 3: present a chronological orders of any change of puncture during the long period of hemodialysis treatment.

d. Document No 4: enables an assessment of the suitability of needles and puncture spots according to the measured arterial and venous pressure.

The documentation is completed by written instructions for patients about their care for AVF and precaution measures to be taken at home in the case of complications.

Such documentation enables the monitoring of any changes of AVF as well as any disturbing of its functioning. So, early diagnostic procedures could be made and if needed early correction (surgical or radiological) could be performed. This documentation provides the new opportunities for a long lasting AVF presentation.

It may also be used for learning a new stuff about AVF of each patient and for practicing of AVF puncture.

\section{6}

The Dialock ${ }^{\circledR}$ Vascular Access System: A New Subcutaneous Port for Hemodialysis

\author{
S. Zitta, M. Hessinger*, K. Tiesenhausen*, P. Krisper, \\ H. Holzer \\ Department of Medicine, Division of Nephrology, \\ *Department of Surgery, Division of Vascular Surgery, \\ University Hospital of Graz, Graz, Austria
}

Background: Vascular access remains a major problem in the delivery of adequate hemodialysis. The arteriovenous fistula is still considered the gold standard for vascular access. However, due to age and co-morbidity the number of hemodialysis patients with central venous catheters (CVC) increases worldwide. International data show that $15 \%$ of vascular access for chronic hemodialysis are central venous catheters. CVCs proposed as an alternative to grafts or fistulas are associated with a significant risk of infection. Therefore a new implantable port system (Dialock $^{\circledR}$, Biolink Corporation, Norwell, MA, USA) has been developed as an innovative approach to vascular access in order to reduce such hazards. The metallic port-like device is implanted subcutaneously in a pocket below the clavicle, and two permanent silicone catheters attached to the device are staggered within the right atrium. Accessed by percutaneous puncture this port provides a linear blood flow passage. During dialysis-free intervals patency of the catheters is ensured by an antithrombotic lock solution (heparin, low-molecular-weight heparin, sodium-citrate, or taurolidin plus citrate).

Methods: From November 1999 to January 2002 Dialock systems were implanted in 16 patients ( 13 males, 3 females, mean age 58 years, range 26-77 years) in our center. In 3 patients with end stage renal disease (ESRD) Dialock system was the first choice vascular access, 13 patients had been on chronic hemodialysis treatment between 2 months and 22 years. Indication for Dialock implantation was dilated cardiomyopathy $(n=9)$, pulmonary hypertension $(n=2)$, vascular access problems with repeated fistula thrombosis $(n=2)$, severe steal syndrome with finger necrosis $(n=1)$, paranoia with repeated withdraws of central venous catheters by the patient $(n=1)$, and severe diabetic micro- and macroangiopathy $(\mathrm{n}=1)$. At the same 
time 10 dialysis patients in our unit received a new central venous catheter.

Results: Dialock was implanted in 16 patients under local anesthesia. The device was accessed for hemodialysis treatment 3.7 days (range 0-7) after implantation. During a period of 26 months we observed the following complications (rate per 1,000 patient days) in Dialock systems (3,500 patient days) compared with CVCs $(1,700$ patient days):

\begin{tabular}{llr}
\hline & Dialock & CVC \\
\hline Skin infections & 0.6 & 12.4 \\
Blood stream infections & 0.6 & 2.9 \\
Clotting & 4.0 & 5.2 \\
\hline
\end{tabular}

There was no difference between Dialock and CVC concerning prescribed blood flow $(250-300 \mathrm{ml} / \mathrm{min})$ for each dialysis session. The recirculation rate was measured by Transonic HD01plus hemodialysis monitor (Transonic Systems Inc., Ithaca, New York)
The Dialock system had a recirculation rate of $0-3 \%$, in CVCs recirculation rate was measured between 2 and $7 \%$. Central venous catheters were filled with heparin. Dialock systems were locked with taurolidin plus citrate $\left(\right.$ Neutrolin $^{\circledR}$, Biolink Corporation, Norwell, MA, USA), a new antimicrobial catheter lock solution. Patients were very satisfied with this new access and reported an increase in quality of life. In 2 patients suffering from dilated cardiomyopathy ejection fraction increased significantly from $20 \%$ to $40 \%$ after Dialock implantation and ligature of the previous AV fistula. Eleven Dialock devices are currently working without any problems. Two ports had to be explanted because of pocket infection. Three patients died unrelated to the device or dialysis treatment.

Conclusion: The Dialock device offers a new very interesting and promising vascular access for ESRD patients who suffer from heart failure, pulmonary hypertension, poor vessels or steal syndrome. Furthermore, our data show distinct lower complication rates compared with central venous catheters. Another very important fact is the clinical improvement of patients with cardiomyopathy and the increase in quality of life in our hemodialysis patients. 


\section{Author Index for Abstracts}

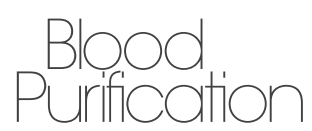

Bakran, A. 14, 26

Barbosa, J. 8, 17, 18

Blankestijn, P.J. 34

Bourquelot, P. 6, 10, 15

Foley, R.N. 29

Hessinger, M. 36

Holzer, H. 36

Huisman, R.M. 34

José, F.M. 8, 17, 18

Numbers refer to Abstract No.

Krisper, P. 36

Krivitski, N.M. 16

Lazarides, M.K. 11

Malovrh, M. 4, 7, 35

Mickley, V. 23, 33

Pecik-Gostincar, M. 35

Pengloan, J. 13, 31

Pogorevc, Z. 35

Polo, J.R. 9, 28
Ponikvar, J.B. 32

Ponikvar, R. 3

Premru, V. 2

Raynaud, A. 5, 25

Semolic, A. 35

Tiesenhausen, K. 36

Tordoir, J.H.M. 12, 20, 34

Turmel-Rodrigues, L. 19, 24 van der Mark, W.A.M.A. 34

van der Sande, F. 34

van Loon, M. 34

Vorwerk, D. 22

Yerdel, M.A. 30

Zijlstra, J.J. 34

Zitta, S. 36

\section{KARGER}

(C) 2002 S. Karger AG, Basel

Fax +4161306 1234

E-Mail karger@karger.ch

www.karger.com 